\title{
Facilitation of hERG channels by blockers: a mechanism predicted to reduce lethal cardiac arrhythmias
}

Authors: Kazuharu Furutani ${ }^{1,2,3, \dagger, *}$, Kunichika Tsumoto ${ }^{1, \dagger}$, I-Shan Chen ${ }^{1}$, Kenichiro Handa ${ }^{1}$, Yuko Yamakawa ${ }^{1}$, Jon T. Sack ${ }^{3}$, Yoshihisa Kurachi ${ }^{1,2, *}$

\author{
Affiliations: \\ ${ }^{1}$ Department of Pharmacology, Graduate School of Medicine, Osaka University, Osaka 565- \\ 0871, Japan. \\ ${ }^{2}$ Center for Advanced Medical Engineering and Informatics, Osaka University, Osaka 565-0871, \\ Japan. \\ ${ }^{3}$ Department of Physiology and Membrane Biology, University of California Davis, Davis, CA \\ 95616, USA.
}

\section{Correspondences:}

Kazuharu Furutani, PhD

Department of Physiology and Membrane Biology, University of California, Davis, 4147 Tupper Hall, One Shields Avenue, Davis, CA 95616, Tel: +1-530-752-2180, E-mail:

kfurutani@ucdavis.edu.

Yoshihisa Kurachi, MD, PhD

Division of Molecular and Cellular Pharmacology, Department of Pharmacology, Graduate School of Medicine, Osaka University, 2-2 Yamada-oka, Suita, Osaka 565-0871, Japan. Tel: +81 66879 3512; Fax: +81 66879 3519. E-mail: ykurachi@pharma2.med.osaka-u.ac.jp

$\dagger$ These authors contributed equally to this work.

Abbreviations: AP: action potential; APD: action potential duration; APD90: action potential duration measured at 90\% repolarization; EAD: early afterdepolarization; hERG: human ether-ago-go-related gene; $I_{\mathrm{CaL}}$ : L-type $\mathrm{Ca}^{2+}$ channel current; $I_{\text {net }}$ : net ionic current; $I_{\mathrm{K} 1}$ : inward-rectifier potassium current; $I_{\mathrm{Kr}}$ : rapid component of the delayed-rectifier potassium current; $I_{\mathrm{Na}}$ : sodium current; ORd: O’Hara-Rudy dynamic 


\begin{abstract}
:
Fatal cardiac arrhythmias are caused by some, but not all, drugs that inhibit the cardiac rapid delayed-rectifier current $\left(I_{\mathrm{Kr}}\right)$ by blocking hERG channels. Here, we propose a novel mechanism that could make certain hERG blockers less proarrhythmic. Several drugs that block hERG channels, yet have favorable cardiac safety profiles, also evoke another effect; they increase the current amplitude upon low-voltage depolarization (facilitation). Voltage-clamp recordings of hERG block and facilitation by nifekalant, a Class III antiarrhythmic agent, constrained a model of human cardiac $I_{\mathrm{Kr}}$. In human ventricular action potential simulations, nifekalant showed its therapeutic ability to suppress ectopic excitations, with or without facilitation. Without facilitation, excessive $I_{\mathrm{Kr}}$ block evoked early afterdepolarizations, which cause lethal arrhythmias. Facilitation prevented early afterdepolarizations at the same degree of block by increasing $I_{\mathrm{Kr}}$ during repolarization phase of action potentials. Thus, facilitation is proposed to reduce the arrhythmogenic risk of hERG blockers.
\end{abstract}




\section{Introduction}

A rapid component of the delayed-rectifier potassium current $\left(I_{\mathrm{Kr}}\right)$ plays an important role in the repolarization of the cardiac action potential (AP). $I_{\mathrm{Kr}}$ is especially important in ventricular muscle of the hearts of large mammals. Certain blockers of $I_{\mathrm{Kr}}$ are class III antiarrhythmic agents, used to treat ventricular tachyarrhythmias (Sanguinetti and Jurkiewicz 1990, Vaughan Williams 1992, Zeng et al. 1995, Clancy et al. 2003). Blockers of $I_{\mathrm{Kr}}$ prolong the AP duration (APD) and effective refractory period (ERP) to suppress premature ventricular contraction. Blockade of $I_{\mathrm{Kr}}$ also is a side-effect of many drugs. Blockers of $I_{\mathrm{Kr}}$ can cause acquired long QT syndrome and the life-threatening ventricular tachyarrhythmia called "torsades de pointes" (Surawicz 1989, Sanguinetti et al. 1995, Roden 2000, Sanguinetti and Tristani-Firouzi 2006, Roden 2008). Emphasis on detecting $I_{\mathrm{Kr}}$ blockade early in drug discovery (ICH 2005) has contributed to the successful removal of torsades de pointes risk for new chemical entities. However, there is concern that promising new drug candidates are being unnecessarily discarded because they benignly block $I_{\mathrm{Kr}}$ (Sager et al. 2014, Gintant et al. 2016). Indeed, numerous examples exist of drugs that block $I_{\mathrm{Kr}}$ and prolong the QT interval but have little proarrhythmic risk (De Ponti et al. 2001, Redfern et al. 2003).

The human ether-a-go-go-related gene (hERG) encodes the ion channel through which $I_{\mathrm{Kr}}$ current passes (Sanguinetti et al. 1995). We have found that some hERG blockers also increase hERG currents at potentials close to the threshold for channel activation. We refer to this electrical phenomenon as 'facilitation' (Hosaka et al. 2007, Furutani et al. 2011, Yamakawa et al. 2012). A series of hERG blockers with lower proarrhythmic risk were found to evoke facilitation of hERG (Hosaka et al. 2007, Furutani et al. 2011, Yamakawa et al. 2012). The correlation between clinical safety and facilitation lead to our current hypothesis: facilitation by hERG blockers increases $I_{K r}$ during cardiac AP repolarization and thereby decreases their proarrhythmic risk. Stringently testing this hypothesis requires a system where facilitation can be selectively removed from a hERG blocker's mechanism. In the present study, we test the aforementioned facilitation hypothesis with a mathematical model of the actions of nifekalant, a hERG channel blocker that induces facilitation and has been used safely in the treatment of lifethreatening ventricular tachyarrhythmias (Nakaya et al. 1993, Takenaka et al. 2001, Igawa et al. 2002, Sato et al. 2017), on the APs of human ventricular myocytes. 


\section{Results}

AP voltage stimuli induce facilitation by nifekalant

The degree of facilitation of $I_{\mathrm{Kr}}$ depends on the dose of hERG blocker and the membrane's history of voltage changes. Facilitation by blockers, with the exception of amiodarone derivative KB130015 (Gessner et al. 2010), requires a preceding strong depolarization as a conditioning stimulus (Carmeliet 1993, Jiang et al. 1999, Hosaka et al. 2007, Furutani et al. 2011, Yamakawa et al. 2012). To test whether cardiac APs are sufficient to stimulate facilitation, we applied an AP clamp protocol to hERG channels. Repeated stimulation with a human ventricular myocyte waveform at $1 \mathrm{~Hz}$ induced facilitation of nifekalant-treated hERG channels (Figure 1). The facilitation effect on hERG current saturated as the number of APs increased, indicating facilitation reached $90 \%$ of its steady state level within 20 heartbeats (Figure 1C). The induction of facilitation by cardiac AP stimuli suggests that facilitation is potentially physiologically relevant.

\section{Facilitation can be incorporated into cardiac electrophysiology modeling}

To understand how facilitation could impact cellular electrophysiology mechanisms specific to human ventricular myocytes, we used the O'Hara-Rudy dynamic (ORd) human ventricular AP model (O'Hara et al. 2011). We modified the $I_{\mathrm{Kr}}$ formula in the ORd model to reproduce hERG channel block by nifekalant, with or without facilitation. This modified $I_{\mathrm{Kr}}$ formula was constrained by currents recorded from HEK cells expressing hERG (Figure 2A-C; Supplemental Figure 1). Figure 2 shows the agreement of our $I_{\mathrm{Kr}}$ model with hERG current from HEK cells and its modulation by nifekalant. Left panels of Figure 2D, E show experimental hERG currents blocked with $100 \mathrm{nM}$ nifekalant, before (magenta traces) and after the induction of facilitation effect by a conditioning pulse (green traces). After the induction of the facilitation effect, nifekalant enhanced the current induced by a $-30 \mathrm{mV}$ pulse (Figure 2D), but it still suppressed the current induced by a $+30 \mathrm{mV}$ pulse (Figure $2 E$ ). The current enhancement is due to a modification of channel activation gating that shifts the voltage dependence to more hyperpolarized membrane potentials (Hosaka et al. 2007, Furutani et al. 2011). The voltage dependence of the hERG current in the facilitation condition could be described as the sum of two Boltzmann functions reflecting two populations of $\mathrm{hERG}$ currents having different 
activation voltage dependences (Furutani et al. 2011). The high- $V_{1 / 2}$ fraction of the channel population has biophysical characteristics typical for the hERG channel, while the low- $V_{1 / 2}$ fraction is 'facilitated'. The facilitated $V_{1 / 2}$ was shifted negative by $-26.5 \mathrm{mV}$ (Table 1 ). This shift was consistent with that of hERG channels expressed in Xenopus oocytes (Furutani et al. 2011). Thus, the facilitation effect could be explained by a fraction of channels opening with a negatively shifted activation curve (Figure $2 F, G$ ). Inclusion of the facilitation effect in the $I_{\mathrm{Kr}}$ model reproduced measurements of hERG currents from voltage-step experiments (Figure 2D$G$ ), and over a range of concentrations (Supplemental Figure 2). In AP clamp experiments and simulations, our $I_{\mathrm{Kr}}$ model also predicted the effects of nifekalant on hERG currents during AP (Figure 2H), indicating that the model describes the block and facilitation effects of nifekalant sufficiently to allow us to assess the impact of facilitation in simulations of human ventricular myocyte electrophysiology.

Facilitation selectively increases $I_{K r}$ during late phase-2 and phase-3 repolarization of cardiac APs

To understand the role of facilitation in the cardiac AP, we investigated the influence of facilitation on AP morphology. When endocardial APs are evoked with $1 \mathrm{~Hz}$ pacing, $100 \mathrm{nM}$ nifekalant prolonged the APD measured at 90\% repolarization, APD90, (334.4 ms, vs. $257.4 \mathrm{~ms}$ in the control condition, see Figure $3 A$ ), in agreement with experimental results in human ventricular myocytes (Jost et al. 2005). The AP was slightly further prolonged in simulations with $I_{\mathrm{Kr}}$ block without facilitation $\left(342.0 \mathrm{~ms}^{\mathrm{APD}}{ }_{90}\right.$, compare green and magenta traces in Figure $3 A$ ). In the model, $100 \mathrm{nM}$ nifekalant blocks $40 \%$ of total $I_{\mathrm{Kr}}$ at voltages $>0 \mathrm{mV}$ with or without facilitation (Figure 2G). When facilitation is implemented in the model, $32 \%$ of the hERG channels enter a facilitated state such that they produce substantially more $I_{\mathrm{Kr}}$ than control conditions when the potential is between -20 and $-50 \mathrm{mV}$ (Figure $2 G$ ). There was little difference between the voltage-dependent activation variables in the $I_{\mathrm{Kr}}$ model during phase- 1 and phase- 2 of the AP ( $x_{\mathrm{r} 1}$ and $x_{\mathrm{r} 2}$ for the unfacilitated channels and facilitated channels, respectively), indicating $I_{\mathrm{Kr}}$ behaved similarly (Figure $3 B, C$ ). During late phase-2 and phase-3 repolarization, an increase can be seen in $I_{\mathrm{Kr}}$ (asterisks in Figure $3 \mathrm{~A}-\mathrm{C}$ ). However, in block conditions with or without facilitation, the APs were terminated by increase in the inward- 
rectifier potassium current $\left(I_{\mathrm{K} 1}\right)$-mediated repolarization current (Figure $3 D$ ) after a short time, before much prolongation of the AP by facilitation. Thus, in this model of a healthy heart under unstressed conditions, facilitation by this hERG blocker selectively increases $I_{\mathrm{Kr}}$ during late phase- 2 and phase- 3 repolarization, but this has only a subtle effect on the cardiac AP.

\section{Facilitation relieves reverse frequency-dependence of APD prolongation}

Class III arrhythmic agents are hERG blockers that are used clinically to suppress ventricular tachyarrhythmias (Sanguinetti and Jurkiewicz 1990, Vaughan Williams 1992, Sanguinetti and Tristani-Firouzi 2006). To suppress tachyarrhythmias without provoking torsades de pointes, $I_{\mathrm{Kr}}$ block would ideally be use-dependent and prolong APD only in response to high frequency stimulation (Surawicz 1989, Hondeghem and Snyders 1990). Previous studies reported that actions of sotalol (Hafner et al. 1988, Hondeghem and Snyders 1990) and dofetilide (Tande et al. 1990), trend against this antiarrhythmic ideal by prolonging APDs more at low than high frequencies, showing reverse frequency-dependence. Notably, sotalol and dofetilide are hERG blockers that do not induce facilitation (Furutani et al. 2011, Yamakawa et al. 2012).

Nifekalant also showed reverse frequency-dependence, but it was not as marked (Nakaya et al. 1993, Cheng et al. 1996, Igawa et al. 2002). To determine why, we used our model to test the frequency-dependent effects of nifekalant on APD. The left panel of Supplemental Figure 3 shows changes in the $\mathrm{APD}_{90}$ with $100 \mathrm{nM}$ nifekalant at stimulation frequencies from 0.2 to $2 \mathrm{~Hz}$. Without facilitation (magenta trace in Supplemental Figure 3, left), the prolongation of APD was more effective at lower stimulation frequencies, resulting in a reverse frequency-dependence. When facilitation was included in the model, the reverse frequency-dependence became slightly weaker at low frequencies (green trace in Supplemental Figure 3, left). APD 90 with facilitation was $5.5 \mathrm{~ms}$ shorter than without at $2.0 \mathrm{~Hz}, 7.7 \mathrm{~ms}$ shorter at $1.0 \mathrm{~Hz}$, and $10.7 \mathrm{~ms}$ shorter at 0.2 Hz (Supplemental Figure 3, right). This suggests facilitation can partially relieve the reverse frequency-dependence that is a risk factor for torsades de pointes. 
Heart failure patients are at risk for malignant ventricular arrhythmias. Clinical and theoretical data has shown that the APs in heart failure patients at slow and modest heart rates are destabilized compared with healthy subjects (Bayer et al. 2010). A more recent study indicated the APs of the heart failure models exhibit stronger rate-dependence when compared with the APs of the normal model, and can generate early afterdepolarizations (EADs) and alternans at modest pacing rates (Elshrif et al. 2015). These features are thought to trigger ventricular arrhythmias (Roden 2000, Thomsen et al. 2003, Nattel et al. 2007, Roden 2008, Weiss et al. 2010). To determine whether facilitation might have a more critical impact on failing hearts, we investigated the frequency-dependent effects of $I_{\mathrm{Kr}}$ facilitation on the simulated AP in a heart failure model (Figure 4) (Elshrif et al. 2015). APs in the heart failure model were prolonged from the control (non-heart failure) model (compare black traces in Figure 4B, Supplemental Figure 3), and were further prolonged by $I_{\mathrm{Kr}}$ blockade. The effect of facilitation was more prominent with low frequency pacing of the heart failure model, and the reverse frequencydependence of APD prolongation was more dramatically attenuated (compare Figure 4A, Supplemental Figure 3, left) because the delayed repolarization at low frequency pacing prolongs the latency before facilitation increases the $I_{\mathrm{Kr}}$ current. APD 90 during block with facilitation was $16.5 \mathrm{~ms}$ shorter than without facilitation at $2.0 \mathrm{~Hz}, 36.3 \mathrm{~ms}$ shorter at $1.0 \mathrm{~Hz}$, and $53.0 \mathrm{~ms}$ shorter at $0.2 \mathrm{~Hz}$ (Figure $4 B$ ), suggesting that the electrical remodeling in failing myocytes makes cellular responses more sensitive to $I_{\mathrm{Kr}}$ modulation.

\section{$I_{\text {Kr facilitation prevents } E A D}$}

Excessive AP prolongation by a hERG blocker showing strong reverse frequency-dependence creates an electrophysiological environment that favors the development of EAD at low stimulating frequencies. Increasing $I_{\mathrm{Kr}}$ block from $40 \%$ to $50 \%$ caused further APD prolongation (Figure $4 C, D$ ). Without facilitation, the APD was dramatically prolonged with low frequency pacing (magenta trace in Figure 4C). We found that in the heart failure model paced at $0.2 \mathrm{~Hz}$, EAD appeared at $50 \% I_{\mathrm{Kr}}$ block without facilitation (magenta traces in Figure 4D), and facilitation suppressed these EADs (green traces in Figure 4D). Although this pacing is unphysiologically slow, these findings suggest hERG facilitation could have a more significant impact on arrhythmogenesis in a failing heart. 
To determine the range of conditions over which facilitation could potentially suppress proarrythmic effects of hERG blockade, we extended analysis in the heart failure model. Figure $5 A-C$ shows examples of steady-state AP trains in several $I_{\mathrm{Kr}}$ block conditions. Figure $5 D$ shows the changes in $\mathrm{APD}_{90}$ as a function of the degree of $I_{\mathrm{Kr}}$ block. Below $40 \%$ block, the drug prolonged $\mathrm{APD}_{90}$ similarly with or without facilitation (Figure 5A, D). Without facilitation, APs were destabilized when $I_{\mathrm{Kr}}$ inhibition was $>48 \%$ (section sign in Figure 5D; $\left.\mathrm{APD}_{90}=890.0 \mathrm{~ms}\right)$, resulting in an alternating EADs and periodic EADs. With facilitation, EADs did not appear until 57\% inhibition of $I_{\mathrm{Kr}}$ (pipes in Figure 5D; APD90 $=896.4 \mathrm{~ms}$ ), indicating that $I_{\mathrm{Kr}}$ facilitation stabilized the AP. Figure $5 B, C$ show examples of steady-state AP trains with $50 \%$ and $55 \%$ block of $I_{\mathrm{Kr}}$ (conditions indicated by dagger and double-dagger in Figure 5D, bottom), respectively. These results suggest the facilitation of $I_{\mathrm{Kr}}$ by blockers reduces proarrhythmic side effects by preventing the development of EADs. In the non-failing heart model, a similar antiarrhythmic effect of facilitation was observed with more extreme $I_{\mathrm{Kr}}$ blockade (Supplemental Figure 4).

$I_{\mathrm{Kr}}$ facilitation suppresses reactivation of L-type $\mathrm{Ca}^{2+}$ channels. In the heart failure model at 55\% inhibition of $I_{\mathrm{Kr}}$ without facilitation (magenta lines in Figure 6, left), the repolarization delay caused by $I_{\mathrm{Kr}}$ reduction augmented the window current in L-type $\mathrm{Ca}^{2+}$ channel current $\left(I_{\mathrm{CaL}}\right)$ during late AP phase-2 with each stimulation (Figure 6C, left). As a result, the evoked APs were gradually prolonged with each stimulation, leading to further augmentation of the $I_{\mathrm{CaL}}$ window current (Figure 6C, left) and AP prolongation (Figure 6B, left). Then, the stagnated repolarization caused more reactivation of L-type $\mathrm{Ca}^{2+}$ channels (arrowhead in Figure 6C, left), enhancing an inward current component of the net ionic current ( $\left.I_{\text {net }}\right)$ during late AP phase-2 (Figure 6D, left). This caused the inward-outward balance of $I_{\text {net }}$ (asterisks in Figure $6 D$, left) that temporally interrupted AP repolarization, followed by a second rising phase of the AP that led to EAD. This $I_{\mathrm{Kr}}$ block-induced dysfunction eventually converged to a periodic EAD response (Figure $5 C$ and Figure $6 A$, right column). In contrast, an increase in $I_{\mathrm{Kr}}$ due to facilitation (Figure $6 E$, right) enhanced an outward current component in $I_{\text {net }}$ during late AP phase-2 and phase-3 (Figure 6D, right). This accelerated the membrane repolarization and completed the AP repolarization (Figure 6B, right). Thus, facilitation suppresses EADs by selectively amplifying $I_{\mathrm{Kr}}$ during late phase-2 and phase-3 of APs when the repolarization is in danger of stagnating. The facilitated $I_{\mathrm{Kr}}$ overwhelms the reactivating $I_{\mathrm{CaL}}$, preventing EAD. 


\section{Modeling suggests $I_{K r}$ facilitation could improve patient safety}

Class III antiarrhythmic agents are powerful antiarrhythmics used to treat patients with serious ventricular tachycardias in spite of their narrow therapeutic index (Tamargo et al. 2015). The maximal therapeutic dose of Class III antiarrhythmics is generally limited by proarrhythmic risk of $I_{\mathrm{Kr}}$ block. At low doses with minimal proarrhythmic risk, nifekalant suppresses malignant ventricular tachyarrhythmia (Takenaka et al. 2001) and improves short-term and longterm survival of adult patients with ventricular fibrillation/pulseless ventricular tachycardia (Sato et al. 2017). In animal models, a torsades de pointes response was not detected with the therapeutic dose of nifekalant (Satoh et al. 2004), and nifekalant has a broader safety window than $I_{\mathrm{Kr}}$ blockers without facilitation (Sugiyama 2008, Yamakawa et al. 2012). To determine whether nifekalant's favorable safety profile could be related to the facilitation mechanism, we conducted simulations of nifekalant's predicted safety window with and without facilitation.

We calculated the "therapeutic" dose of nifekalant that prolongs APD 90 by $500 \mathrm{~ms}$, an established clinical standard (Igawa et al. 2002, Drew et al. 2010). With facilitation, the simulated therapeutic dose was $278.9 \mathrm{nM}$ (68.0\% $I_{\mathrm{Kr}}$ block); without facilitation, 14\% less drug was required, $240.2 \mathrm{nM}\left(64.1 \% I_{\mathrm{Kr}}\right.$ block) (Figure 7). To confirm the antiarrhythmic effect of nifekalant at those therapeutic doses, we assessed the vulnerability of cardiomyocytes to premature excitation. We measured the refractory period of APs in the normal, non-failing model by calculating the shortest timing sufficient to produce secondary voltage overshoot by a second stimuli (S2 stimuli) after regular pacing (S1-S2 stimulation protocol). The effective refractory periods with nifekalant were $216 \mathrm{~ms}$ or $215 \mathrm{~ms}$ longer than control, with or without facilitation, respectively (Supplemental Figure 5), suggesting that facilitation has little impact on effectiveness as a Class III antiarrhythmic. We simulated the safety window for nifekalant as the ratio of the dose that produces toxicity (EAD) to the therapeutic dose. Without facilitation, EADs occurred at $657.5 \mathrm{nM}, 2.74 \times$ the calculated therapeutic dose (Figure 7). With facilitation, EADs did not occur until $894.5 \mathrm{nM}, 3.21 \times$ the therapeutic dose. Thus facilitation widens the simulated safety window for EADs, suggesting that with facilitation an $I_{\mathrm{Kr}}$ blocker can remain an efficacious class III antiarrhythmic agent, with an improved safety profile. 


\section{Discussion}

The objective of this study was to examine the influence of the facilitation mechanism on electrical activity in cardiomyocytes. This mathematical model represents a general view of the role that facilitation may play with $I_{\mathrm{Kr}}$ blockers. We found that $I_{\mathrm{Kr}}$ facilitation effects on simulated cardiac APs depended on the AP morphology and APD. Facilitation enhances $I_{\mathrm{Kr}}$ during prolonged APs. The repolarizing effect of facilitated $I_{\mathrm{Kr}}$ is augmented at low frequencies and as APD prolongs, thus avoiding repolarization reserve impairment, and suppressing EAD development. Therefore, hERG channel blockers with facilitation may offer a safety advantage for arrhythmia treatment.

Our conclusions are based on modeling, yet suggest a general cardioprotective mechanism. To exert cardioprotective effects in vivo, certain conditions are required. First, the concentration of the agent needs to reach the effective concentration for facilitation. Second, the membrane potential must induce the $I_{\mathrm{Kr}}$ facilitation effect. We have previously reported the concentration- and voltage-dependence relationships between block and facilitation for several drugs in hERG channels expressed in Xenopus oocytes (Furutani et al. 2011, Yamakawa et al. 2012). In the present study, we evaluated the concentration-dependence of nifekalant on hERG channels expressed in HEK293 cells. As in oocytes, the $\mathrm{IC}_{50} / \mathrm{EC}_{50}$ for block and facilitation by nifekalant were similar $(92.84 \pm 7.71 \mathrm{nM}$ for facilitation; $144.92 \pm$ $16.00 \mathrm{nM}$ for block, see Table 1). However, for other class III antiarrhythmic agents, the concentration-dependences of block and facilitation are distinct. For example, the $\mathrm{EC}_{50}$ for $I_{\mathrm{Kr}}$ facilitation by amiodarone is lower than that for $\mathrm{IC}_{50}$ block (Furutani et al. 2011). These data indicate that when these agents are administrated in the treatment of arrhythmias concentrations effective for block, they certainly also reach the effective concentration for facilitation. Our experimental data showed that facilitation by nifekalant could be induced by cardiac APs (Figure 1). An important finding of this work is that facilitation had a negligible impact on APs under normal conditions (Figure 3), suggesting that facilitation would not greatly affect normal ECGs. Our simulation study suggests that facilitation may have a selective impact during severe repolarization impairment and heart failure conditions, leading to a lower risk of arrhythmias.

It is widely accepted that $I_{\mathrm{Kr}}$ plays an important role in the repolarization of the AP (Surawicz 1989, Sanguinetti and Jurkiewicz 1990, Zeng et al. 1995, Clancy et al. 2003, Sanguinetti and Tristani-Firouzi 2006). The decrease in $I_{\mathrm{Kr}}$ is believed to be associated with 
EADs, an important cause of lethal ventricular arrhythmias in LQT syndrome and heart failure. Experiments from Guo et al (Guo et al. 2011), in isolated non-failing human endocardial ventricular myocytes, showed EADs in the presence of the $I_{\mathrm{Kr}}$ blocker dofetilide $(0.1 \mu \mathrm{M}$, corresponding to $\sim 85 \% I_{\mathrm{Kr}}$ block (Thomsen et al. 2003)), which induces torsades de pointes arrhythmias (Thomsen et al. 2003). In the present study using the modified ORd model, we successfully reproduced the experimental results of Guo et al (Guo et al. 2011), as did the original ORd model (O'Hara et al. 2011).

Reverse-frequency dependent action on APs is a property common to Class III antiarrhythmic agents (Hafner et al. 1988, Hondeghem and Snyders 1990, Tande et al. 1990, Carmeliet 1993, Jurkiewicz and Sanguinetti 1993, Nakaya et al. 1993, Jiang et al. 1999), and the associated proarrhythmic risk limits their clinical usefulness (Hondeghem and Snyders 1990, Okada et al. 1996). Reverse-frequency dependence of $I_{\mathrm{Kr}}$ block was first explained by an increase in the slowly activated delayed-rectifier $\mathrm{K}^{+}$current with rapid heart rates (Jurkiewicz and Sanguinetti 1993). Drug mechanisms can prolong APD at higher stimulation frequencies to attenuate reverse-frequency dependence (Hondeghem and Snyders 1990). For example, some $I_{\mathrm{Kr}}$ blockers, such as vesnarinone, have high affinities to opened and/or inactivated hERG channels and exhibit an accumulation of inhibition at higher stimulation frequencies (usedependent block), reducing proarrhythmic risk (Toyama et al. 1997). In our model, $I_{\mathrm{Kr}}$ block is not altered by stimulation frequency. Therefore, the reverse-frequency dependence may be even less than predicted. Facilitation attenuates reverse-frequency dependence by enhancing $I_{\mathrm{Kr}}$ during late phase-2 and phase-3. The effect on reverse-frequency dependence becomes more prominent at higher concentrations of drug. Figure $4 A$ and Supplemental Figure 3 shows the effects of the modest $I_{\mathrm{Kr}}$ block by $100 \mathrm{nM}$ nifekalant ( 40\% $I_{\mathrm{Kr}}$ block and $\sim 32 \% I_{\mathrm{Kr}}$ facilitation). When the drug concentration increased, the effect of facilitation was more prominent (Figure $4 B$ ) because of the concentration-dependent increase in the facilitated fraction and the prolonged phase 2-3 of AP. Under the higher concentrations of blocker, EADs and alternans emerged at low frequencies that dramatically affect APD (Figure 4B). These EADs were effectively suppressed by $I_{\mathrm{Kr}}$ facilitation (Figure 5, 6, Supplemental Figure 4).

Previous studies showed that $I_{\mathrm{CaL}}$ reactivation is crucial for the development of EADs (Zeng and Rudy 1995, O'Hara et al. 2011, Tsumoto et al. 2017). Prolongation of the time at plateau voltages caused by $I_{\mathrm{Kr}}$ block allows $I_{\mathrm{CaL}}$ reactivation (O'Hara et al. 2011). The EAD 
initiation mechanism in the present study is consistent with an $I_{\mathrm{CaL}}$ reactivation mechanism (Figure 6). $\quad I_{\mathrm{Kr}}$ facilitation accelerates the repolarization just before $I_{\mathrm{CaL}}$ reactivation (Figure 6). Therefore, the inward-outward balance of $I_{\text {net }}$ during the plateau and AP phase- 3 is one determinant for the development of EADs. Such inward-outward balance of $I_{\text {net }}$ results from the interaction of ionic mechanisms underlying the cardiac AP. In addition to an increase in $I_{\mathrm{Kr}}$ by facilitation, these secondary changes may contribute to preventing $I_{\mathrm{CaL}}$ reactivation and development of EADs.

Our modeling suggests that $I_{\mathrm{Kr}}$ facilitation decreases proarrhythmic risk. In considering the clinical and physiological relevance of facilitation to Class III antiarrhythmic agents, it is important to be aware of the limitations of our study. We have not yet examined the facilitation effect on native tissues. Therefore, the absolute voltage- and concentration-dependence of facilitation has not yet been determined with native $I_{\mathrm{Kr}}$ currents. Additionally, off-target effects of blocker may cause further changes, although appreciable cardiac off-target effects of nifekalant have not yet been found.

These limitations notwithstanding, this study sheds light on the potential impact of facilitation on cardiac safety, defining a novel mechanism that could suppress proarrhythmic risk and broaden the safety window of hERG blocking agents. The facilitation effect may explain the reason many clinically useful drugs, especially Class III antiarrhythmic agents, are surprisingly safe, despite hERG block and QT interval prolongation. Meanwhile, a lack of a facilitation effect may explain why dofetilide, d-sotalol, atenolol, and terfenadine have a high risk for lethal arrhythmia (Furutani et al. 2011, Yamakawa et al. 2012). Recent studies have described the general utility of the in silico integration of drug effects on multiple cardiac currents to reduce false-positive and false-negative classifications based on hERG block alone (Mirams et al. 2011, Kramer et al. 2013). More elaborate models incorporating detailed descriptions of ion channel modulation will improve future investigations on the clinical significance of Class III antiarrhythmic agents.

In conclusion, hERG blockers with facilitation effects may have a lower risk for inducing EADs and other triggered activities and thus be more suitable to treat arrhythmias. This finding has the potential to improve the early assessment of cardiotoxicity risk using in vitro ion channel 
bioRxiv preprint doi: https://doi.org/10.1101/341875; this version posted June 8, 2018. The copyright holder for this preprint (which was not certified by peer review) is the author/funder. All rights reserved. No reuse allowed without permission.

assays, thereby reducing the likelihood of mistakenly discarding viable drug candidates and speeding the progression of safer drugs into clinical trials and clinical use. 


\section{Materials and Methods}

\section{Cell preparation and $h E R G$ channel current recording}

A human embryonic kidney (HEK) 293 cell line stably-expressing hERG was kindly provided by Dr. Craig T. January (Zhou et al. 1998). Cells used for electrophysiological study were cultured on coverslips in 12-well plates and transferred to a small recording chamber mounted on the stage of an inverted microscope (Axiovert S100, Carl Zeiss, Oberkochen, Germany), and were superfused with HEPES-buffered Tyrode solution containing (in $\mathrm{mM}$ ) $137 \mathrm{NaCl}, 4 \mathrm{KCl}$, $1.8 \mathrm{CaCl}_{2}, 1 \mathrm{MgCl}_{2}, 10$ glucose, and $10 \mathrm{HEPES}$ ( $\mathrm{pH} 7.4$ with $\mathrm{NaOH}$ ). Membrane currents were recorded in a whole-cell configuration using suction pipettes (Hamill et al. 1981). Leak compensation was not used. The borosilicate micropipette had a resistance of 2-4 M $\Omega$ when filled with the internal pipette solution contained (in $\mathrm{mM}$ ) $120 \mathrm{KCl}, 5.374 \mathrm{CaCl}_{2}, 1.75 \mathrm{MgCl}_{2}, 10$ EGTA, 10 HEPES (pH 7.2 with $\mathrm{KOH}$ ). Liquid junction potential with this internal solution was less than $-4 \mathrm{mV}$, and the off-set was not corrected. Series resistance was typically under 5 $\mathrm{M} \Omega$. Series resistance compensation was used when needed to constrain voltage error to $<10$ $\mathrm{mV}$. Whole-cell recordings were performed using an Axopatch 200B patch-clamp amplifier (Molecular Devices, Sunnyvale, CA), ITC-18 interface and PatchMaster software (HEKA Elektronik, Lambrecht, Germany). The data were stored on a computer hard disk and analyzed using PatchMaster and Igor Pro 7 (WaveMetrics, Portland, OR). In AP Clamp experiments, we used the human ventricular AP waveform simulated with our AP model as voltage-clamp commands. Most experiments were performed at a temperature of $37.0^{\circ} \mathrm{C}$, which was maintained with a CL-200A temperature controller (Warner Instruments, Hamden, CT). In a few experiments, data were initially obtained with HEK293 cell and Xenopus laevis oocyte at room temperature as described previously (Hosaka et al. 2007, Furutani et al. 2011). Frogs (Xenopus laevis) were treated under the guidelines for laboratory animals of Osaka University Graduate School of Medicine.

Nifekalant was obtained from Nihon Schering (Osaka, Japan) and Cayman Chemical (Ann Arbor, MI).

MiRP1 (KCNE2) is a $\beta$-subunit of $I_{\mathrm{Kr}}$ (Abbott et al. 1999). We examined whether nifekalant caused distinct effects on currents mediated by hERG alone and by hERG and KCNE2. In these experiments, we co-expressed KCNE2 in hERG-expressing HEK293 cells by using 
pcDNA3 plasmid vector. However, the drugs caused virtually identical effects on the two channels currents in the dose-response (data not shown) and shift of the activation curve (Table 1). Therefore, we utilized HEK cells and oocytes expressing hERG channels alone for further experiments.

\section{Formulations of kinetic properties for hERG current}

To model the macroscopic current of hERG channels expressed in HEK293 cells, we first estimated the kinetics of the channels. The voltage-dependent activation kinetics (time constant of activation) was determined from current activation experiments (Figure 2A). The activation time constant was measured by fitting $I_{\mathrm{Kr}}$ activation at each depolarized voltage pulse, $V_{\text {depo, }}$ (typically $V_{\text {depo }}$ greater than $-40 \mathrm{mV}$ ) with a single exponential function:

$$
I_{\text {act }}(t)=K\left(1-\exp \left(-t / \tau_{\mathrm{xr}}\left(V_{\mathrm{depo}}\right)\right)\right)
$$

where $K$ is the asymptote and $\tau_{\mathrm{xr}}\left(V_{\text {depo }}\right)$ is the activation time constant. Figure $2 A$ and Supplemental Figure $1 \mathrm{~A}$ shows the activation time constants as a function of $V_{\text {depo. }}$ Furthermore, the voltage-dependent deactivation kinetics was divided into two processes (i.e., fast and slow processes) in accordance with the O'Hara-Rudy formalism (O'Hara et al. 2011). From current deactivation experiments (Supplemental Figure 1B), the deactivation time constants were measured by fitting $I_{\mathrm{Kr}}$ deactivation at each repolarized voltage pulse, $V_{\text {repo }}$, (typically $V_{\text {repo }}$ less than $-30 \mathrm{mV}$ ) to a double exponential function:

$$
I_{\text {deact }}(t)=K+A_{1} \cdot \exp \left(-t / \tau_{\text {xr,fast }}\left(V_{\text {repo }}\right)\right)+A_{2} \cdot \exp \left(-t / \tau_{\text {xr,slow }}\left(V_{\text {repo }}\right)\right),
$$

where $K$ is the asymptote, and $A_{1}$ and $A_{2}$ are the relative components of the fast and slow processes, $\tau_{\mathrm{xr} \text {,fast }}$ and $\tau_{\mathrm{xr} \text {,slow }}$ are the fast and slow deactivation time constants, respectively. Supplemental Figure 1B, C show the fast and slow deactivation time constants as a function of $V_{\text {repo, }}$, respectively. Each time constant value in Eqs. 1 and 2 was determined by nonlinear leastsquares fitting. From these experimental data, the activation and deactivation time constants were expressed as continuous functions of membrane potential, $V_{\mathrm{m}}$, as follows (Supplemental Figure $1 E, F)$ :

For $\tau_{\mathrm{xr}, \text { fast}}$ 


$$
\tau_{\mathrm{xr}, \text { fast }}=\frac{1400}{1+\exp \left(\frac{V_{\mathrm{m}}+10.0}{13.8}\right)}+1300 \cdot\left[\frac{1}{1+\exp \left(-\frac{V_{\mathrm{m}}+55.0}{12.3}\right)}-1\right]
$$

For $\tau_{\mathrm{xr}, \text { slow }}$, if $V_{\mathrm{m}} \geq-80 \mathrm{mV}$

$$
\begin{aligned}
\tau_{\text {xr,slow }}= & \frac{5000}{1+\exp \left(\frac{V_{m}+22.8}{11.5}\right)}+\frac{2000}{1+\exp \left(\frac{V_{m}+25}{2.5}\right)} \\
& +\frac{4000}{1+\exp \left(-\frac{V_{m}+61.5}{5.0}\right)}+\frac{3000}{1+\exp \left(-\frac{V_{m}+54}{2.5}\right)}-7000
\end{aligned}
$$

and $V_{\mathrm{m}}<-80 \mathrm{mV}$,

$$
\tau_{\mathrm{xr}, \mathrm{slow}}=2000+\frac{8000}{1+\exp \left(-\frac{V_{m}+74.9}{10.2}\right)}
$$

\section{Modeling of $I_{K r}$}

The $I \mathrm{Kr}$ current was defined as

$$
I_{\mathrm{Kr}}=G_{\mathrm{Kr}} \cdot O_{\mathrm{p}} \cdot R_{\mathrm{Kr}} \cdot\left(V_{\mathrm{m}}-E_{\mathrm{K}}\right)
$$

where $G_{\mathrm{Kr}}$ is $I_{\mathrm{Kr}}$ conductance $\left(\mathrm{mS} / \mathrm{cm}^{2}\right)$ under the drug action, $V_{\mathrm{m}}$ is the membrane potential $(\mathrm{mV}), E_{\mathrm{K}}$ is the reversal potential, $O_{\mathrm{p}}$ is the open state variable in the activation of $I_{\mathrm{Kr}}$ channel and $R_{\mathrm{Kr}}$ is a time-independent function related to the inactivation property for $I_{\mathrm{Kr}}$, respectively. The voltage-dependence of the $O_{\mathrm{p}}$ in Eq. 6 was defined as

$$
O_{\mathrm{p}}=f \cdot x_{\mathrm{r} 1}+(1-f) \cdot x_{\mathrm{r} 2}
$$

where $x_{\mathrm{r} 1}$ and $x_{\mathrm{r} 2}$ are voltage-dependent activation variables for unfacilitated and facilitated components in the $I_{\mathrm{Kr}}$, respectively, and $f$ is a fraction of unfacilitated component in $I_{\mathrm{Kr}}$ current. The does-fraction relationship of $f$ for nifekalant with an $\mathrm{EC}_{50}$ of $92.84 \mathrm{nM}$ and a Hill coefficient $\left(h_{\mathrm{e}}\right)$ of 1.50 was represented as follows: 


$$
f=\frac{1}{1+\left(\frac{[D]}{\mathrm{EC}_{50}}\right)^{h_{\mathrm{e}}}} .
$$

In O'Hara-Rudy dynamic (ORd) model (O'Hara et al. 2011), the voltage-dependent activation variable was comprised of two variables as follows:

$$
x_{\mathrm{ri}}=A_{\mathrm{xr} \text {,fast }} \cdot x_{\mathrm{ri}, \text { fast }}+A_{\mathrm{xr} \text {,slow }} \cdot x_{\mathrm{r} i \text {,slow }}
$$

where $i$ represent the voltage-dependent type, where the voltage-dependent type can be unfacilitated $\left(x_{\mathrm{r} 1}\right)$ or facilitated $\left(x_{\mathrm{r} 2}\right)$ activation variables, and $A_{\mathrm{xr} \text {,fast }}$ and $A_{\mathrm{xr}, \text { slow }}\left(\equiv 1-A_{\mathrm{xr}, \text { fast }}\right)$ are the fraction of channels with $I_{\mathrm{Kr}}$ activation gate undergoing fast and slow process, respectively. The fraction $A_{\text {xr,fast }}$ was obtained as a following function that best reproduces voltage clamp experimental data (Figure 2):

$$
A_{\text {xr,fast }}=0.05+\frac{0.95}{1+\exp \left(\frac{V_{\mathrm{m}}+73.4}{16.0}\right)} \text {. }
$$

The unfacilitated $\left(x_{\mathrm{r} 1}\right)$ and facilitated activation $\left(x_{\mathrm{r} 2}\right)$ variables were calculated with the following first-order differential equation:

$$
\mathrm{d} x_{\mathrm{r} i, j} / \mathrm{d} t=\Phi \cdot\left(x_{\mathrm{r} i, \infty}-x_{\mathrm{r} i, j}\right) / \tau_{\mathrm{xr}, j}
$$

where $x_{\mathrm{r} i, \infty}$, for $i=1,2$, is the steady-state value of $x_{\mathrm{r} i, j}$, i.e., the voltage-dependent activation curve in the hERG channel, and $\tau_{\mathrm{xr}, j}$ for $j=$ fast, slow, is the time constant for $x_{\mathrm{r} i, j}$, and $\Phi$ is a temperature coefficient as expressed at $3^{((\mathrm{T}-25) / 10)}$, where $\mathrm{T}$ is temperature. The voltage-dependent activation curve in the hERG channel $\left(x_{\mathrm{r} i, \infty}\right.$, for $\left.i=1,2\right)$ is well represented by a single Boltzmann function. Based on our experimental data (Figure $2 A$ and $C$ ), we set the Boltzmann function's half-activation voltage $\left(\mathrm{V}_{1 / 2}\right)$ and the slope factor to $-10.7 \mathrm{mV}$ and of 7.4 , respectively, as the voltage-dependence of the unfacilitated component $\left(x_{\mathrm{r} 1, \infty}\right)$ in $I_{\mathrm{Kr}}$ activation, and shifted the voltage-dependence of facilitated components $\left(x_{\mathrm{r} 2, \infty}\right)$ in $I_{\mathrm{Kr}}$ activation by $26.5 \mathrm{mV}$ in the negative direction (Figure $2 G$ ), i.e.,

$$
x_{\mathrm{r} 1, \infty}=\frac{1}{1+\exp \left(-\frac{V_{\mathrm{m}}+10.7}{7.4}\right)},
$$




$$
x_{\mathrm{r} 2, \infty}=\frac{1}{1+\exp \left(-\frac{V_{\mathrm{m}}+37.2}{7.7}\right)} .
$$

Furthermore, the $R_{\mathrm{Kr}}$ was reconstructed as a following function that best reproduces voltage clamp experimental data (Figure $2 A)$ :

$$
R_{\mathrm{Kr}}=\left[0.12+\frac{0.88}{1+\exp \left(\frac{V_{\mathrm{m}}+60.3}{27}\right)}\right] \cdot\left[0.32+\frac{0.68}{1+\exp \left(\frac{V_{\mathrm{m}}-7}{20}\right)}\right] .
$$

Based on our experimental measurement (Figure 2; Supplemental Figure 2), the $G_{\mathrm{Kr}}$ of when the $I_{\mathrm{Kr}}$ was blocked by nifekalant in a concentration-dependent manner with an $\mathrm{IC}_{50}$ of 144.92 $\mathrm{nM}$ and a Hill coefficient $\left(h_{\mathrm{i}}\right)$ of 1.15 was represented as follows:

$$
G_{\mathrm{Kr}}=g_{\mathrm{Kr}} \cdot \frac{1}{1+\left(\frac{[D]}{\mathrm{IC}_{50}}\right)^{h_{\mathrm{i}}}},
$$

where $[D]$ is the drug concentration $(\mu \mathrm{M})$ and $g_{\mathrm{Kr}}$ is the $I_{\mathrm{Kr}}$ channel conductance absent drug. The $g_{\mathrm{Kr}}$ was set to $0.058 \mathrm{mS} / \mathrm{cm}^{2}$ to recapitulate the APD represented in the ORd model (O'Hara et al. 2011).

In addition, we constructed a conventional block model modified only in $I_{\mathrm{Kr}}$ conductance (block without facilitation model) for comparison with the block model with facilitation.

\section{Simulation protocol and computation}

Voltage-clamping simulations for calculating $I_{\mathrm{hERG}}$ for each clamped voltage pulse with $4 \mathrm{~s}$ duration (Figure 2) were performed using a homemade $\mathrm{C}$ language program, simulating by the forward Euler method with a $0.01 \mathrm{~ms}$ time step. The activation variables $\left(x_{\mathrm{r} i}\right.$, for $\left.i=1,2\right)$ was set equal to zero as an initial condition.

In AP simulations, the $I_{\mathrm{Kr}}$ model in the ORd model was replaced with our experimental based $I_{\mathrm{Kr}}$ model. The simulated AP was calculated by the fourth-order Runge-Kutta method with the double precision numbers. To minimize transient responses in each simulation, pacing 
stimuli of threefold diastolic threshold and the stimuli were applied repeated until the AP response observed in the ORd model reached the stationary state. To evaluate the effects of hERG channel blockade on the vulnerability of a cardiomyocyte to premature ventricular contractions, additional simulations were performed using the S1-S2 stimulation protocol: S1 stimuli until the AP converged to a steady-state were applied at the stimulating frequency of 1 $\mathrm{Hz}$ followed by an S2 stimulus with various coupling intervals. When the arrhythmogenicity of the hERG channel blocker was examined, the stimulating frequency was set to $0.5 \mathrm{~Hz}$ to avoid the stimulus being applied prior to the complete repolarization of the AP. All simulations were encoded in $\mathrm{C} / \mathrm{C}++$, and run on an IBM-compatible computer with the Intel ICC compiler version 15.0.1.

\section{Data and materials availability}

These modified ORd models (non-heart failure and heart failure models) that constructed in the present study were also implemented in an XML-based Physiological Hierarchy Markup Language (PHML), which is available at http://physiolodesigner.org/ as an open-access resource. Details on the modified ORd models as non-heart failing (and failing) endocardial myocytes with and without facilitation effect can referred from PHML models in PH database (https://phdb.unit.oist.jp/modeldb/; ID931 to 936). In addition, all AP simulations presented in this study can be reproduced by performing their PHML model simulations used a software, Flint (http://www.physiodesigner.org/simulation/flint/).

Acknowledgments: We are grateful to Dr. M.T. Keating and Dr. M.C. Sanguinetti (University of Utah) for providing us with hERG clone and Craig T. January (University of Wisconsin) for providing us with HEK293 cell lines stably expressing hERG. We also thank Dr. Colleen Clancy and Dr. Eleonora Grandi (University of California Davis) for discussion. This study was supported by the Hiroshi and Aya Irisawa Memorial Promotion Award for Young Physiologists (to K.F. and K.T.) from the Physiological Society of Japan, Dean Award from Department of Physiology and Membrane Biology, University of California, Davis (to K.F. and J.T.S.), Grants-in-Aid for the Scientific Research on Innovative Areas 22136002 (to Y.K.), 15H01404 (to K.F.), the Scientific Research (C) 15K08231 (to K.F.), 16KT0194 (to K.T.) from 
the Ministry of Education, Science, Sports and Culture of Japan, the Japan Society for the

Promotion of Science, and NIH grants U01HL126273 and R01HL128537 (to K.F. and J.T.S.).

Author contributions: K.F., K.T. and Y.K. designed the experiment; K.F., K.T., I-S.C., K. H., Y. Y. conducted the experiments; K.F. and K.T. analyzed the data; and K.F., K.T., I-S.C., J.T.S., and Y.K. wrote the manuscript. All the authors revised the manuscript.

Competing interests: The authors declare no competing financial interests.

\section{References:}

Abbott GW, Sesti F, Splawski I, Buck ME, Lehmann MH, Timothy KW, Keating MT and Goldstein SA. 1999. MiRP1 forms IKr potassium channels with HERG and is associated with cardiac arrhythmia. Cell 97:175-187. doi:10.1016/S0092-8674(00)80728-X, PMID: 10219239.

Bayer JD, Narayan SM, Lalani GG and Trayanova NA. 2010. Rate-dependent action potential alternans in human heart failure implicates abnormal intracellular calcium handling. Heart Rhythm 7:1093-1101. doi:10.1016/j.hrthm.2010.04.008, PMID: 20382266.

Carmeliet E. 1993. Use-dependent block and use-dependent unblock of the delayed rectifier $\mathrm{K}+$ current by almokalant in rabbit ventricular myocytes. Circulation Research 73:857-868. doi:10.1161/01.RES.73.5.857, PMID: 8403256.

Cheng J, Kamiya K, Kodama I and Toyama J. 1996. Differential effects of MS-551 and E-4031 on action potentials and the delayed rectifier $\mathrm{K}+$ current in rabbit ventricular myocytes. Cardiovascular Research 31:963-974, PMID: 8759253.

Clancy CE, Kurokawa J, Tateyama M, Wehrens XH and Kass RS. 2003. K+ channel structureactivity relationships and mechanisms of drug-induced QT prolongation. Annual Review of Pharmacology and Toxicology 43:441-461. doi:10.1146/annurev.pharmtox.43.100901.140245, PMID: 12540747.

De Ponti F, Poluzzi E and Montanaro N. 2001. Organising evidence on QT prolongation and occurrence of Torsades de Pointes with non-antiarrhythmic drugs: a call for consensus. European Journal of Clinical Pharmacology 57:185-209. doi:10.1007/s002280100290, PMID: 11497335.

Drew BJ, Ackerman MJ, Funk M, Gibler W, Klingfield P, Menon V, Philippides GJ, Roden DM and Zareba W. 2010. Prevention of Torsade de Pointes in Hospital Settings: A Scientific Statement From the American Heart Association and the American College of Cardiology Foundation. Circulation 121:1047-1060. doi:10.1161/CIRCULATIONAHA.109.192704, PMID: 20185054.

Elshrif MM, Shi P and Cherry EM. 2015. Representing variability and transmural differences in a model of human heart failure. IEEE J Biomed Health Inform 19:1308-1320. doi:10.1109/JBHI.2015.2442833, PMID: 26068919. 
Furutani K, Yamakawa Y, Inanobe A, Iwata M, Ohno Y and Kurachi Y. 2011. A mechanism underlying compound-induced voltage shift in the current activation of $\mathrm{hERG}$ by antiarrhythmic agents. Biochemical and Biophysical Research Communications 415:141146. doi:10.1016/j.bbrc.2011.10.034, PMID: 22020101.

Gessner G, Macianskiene R, Starkus JG, Schonherr R and Heinemann SH. 2010. The amiodarone derivative KB130015 activates hERG1 potassium channels via a novel mechanism. European Journal of Pharmacology 632:52-59. doi:10.1016/j.ejphar.2010.01.010, PMC2835770.

Gintant G, Sager PT and Stockbridge N. 2016. Evolution of strategies to improve preclinical cardiac safety testing. Nature Reviews Drug Discovery 15:457-471. doi:10.1038/nrd.2015.34, PMID: 26893184.

Guo D, Liu Q, Liu T, Elliott G, Gingras M, Kowey PR and Yan GX. 2011. Electrophysiological properties of HBI-3000: a new antiarrhythmic agent with multiple-channel blocking properties in human ventricular myocytes. Journal of Cardiovascular Pharmacology 57:79-85. doi:10.1097/FJC.0b013e3181ffe8b3, PMID: 20980921.

Hafner D, Berger F, Borchard U, Kullmann A and Scherlitz A. 1988. Electrophysiological characterization of the class III activity of sotalol and its enantiomers. New interpretation of use-dependent effects. Arzneimittel-Forschung 38:231-236, PMID: 3370070.

Hamill OP, Marty A, Neher E, Sakmann B and Sigworth FJ. 1981. Improved patch-clamp techniques for high-resolution current recording from cells and cell-free membrane patches. Pflügers Archiv - European Journal of Physiology 391:85-100. doi:10.1007/BF00656997, PMID: 6270629.

Hondeghem LM and Snyders DJ. 1990. Class III antiarrhythmic agents have a lot of potential but a long way to go. Reduced effectiveness and dangers of reverse use dependence. Circulation 81:686-690. doi:10.1161/01.CIR.81.2.686, PMID: 2153477.

Hosaka Y, Iwata M, Kamiya N, Yamada M, Kinoshita K, Fukunishi Y, Tsujimae K, Hibino H, Aizawa Y, Inanobe A, Nakamura H and Kurachi Y. 2007. Mutational analysis of block and facilitation of HERG current by a class III anti-arrhythmic agent, nifekalant. Channels (Austin) 1:198-208. doi:10.4161/chan.4691, PMID: 18690032.

ICH. 2005. The non-clinical evaluation of the potential for delayed ventricular repolarization (QT interval prolongation) by human pharmaceuticals. Guidance on $S 7 B$.

Igawa M, Aonuma K, Okamoto Y, Hiroe M, Hiraoka M and Isobe M. 2002. Anti-arrhythmic Efficacy of Nifekalant Hydrochloride, a Pure Class III Anti-arrhythmic Agent, in Patients With Healed Myocardial Infarction and Inducible Sustained Ventricular Tachycardia. Journal of Cardiovascular Pharmacology 40:735-742, PMID: 12409982.

Jiang M, Dun W, Fan JS and Tseng GN. 1999. Use-dependent 'agonist' effect of azimilide on the HERG channel. Journal of Pharmacology and Experimental Therapeutics 291:13241336, PMID: 10565858.

Jost N, Virag L, Bitay M, Takacs J, Lengyel C, Biliczki P, Nagy Z, Bogats G, Lathrop DA, Papp JG and Varro A. 2005. Restricting excessive cardiac action potential and QT prolongation: a vital role for IKs in human ventricular muscle. Circulation 112:13921399. doi:10.1161/CIRCULATIONAHA.105.550111, PMID: 16129791.

Jurkiewicz NK and Sanguinetti MC. 1993. Rate-dependent prolongation of cardiac action potentials by a methanesulfonanilide class III antiarrhythmic agent. Specific block of rapidly activating delayed rectifier K+ current by dofetilide. Circulation Research 72:7583. doi:10.1161/01.RES.72.1.75, PMID: 8417848. 
Kramer J, Obejero-Paz CA, Myatt G, Kuryshev YA, Bruening-Wright A, Verducci JS and Brown AM. 2013. MICE models: superior to the HERG model in predicting Torsade de Pointes. Scientific Reports 3:2100. doi:10.1038/srep02100, PMID: 23812503.

Mirams GR, Cui Y, Sher A, Fink M, Cooper J, Heath BM, McMahon NC, Gavaghan DJ and Noble D. 2011. Simulation of multiple ion channel block provides improved early prediction of compounds' clinical torsadogenic risk. Cardiovascular Research 91:53-61. doi:10.1093/cvr/cvr044, PMID: 21300721.

Nakaya H, Tohse N, Takeda Y and Kanno M. 1993. Effects of MS-551, a new class III antiarrhythmic drug, on action potential and membrane currents in rabbit ventricular myocytes. British Journal of Pharmacology 109:157-163. doi:10.1111/j.14765381.1993.tb13546.x, PMID: 7684298.

Nattel S, Maguy A, Le Bouter S and Yeh YH. 2007. Arrhythmogenic ion-channel remodeling in the heart: heart failure, myocardial infarction, and atrial fibrillation. Physiological Reviews 87:425-456. doi:10.1152/physrev.00014.2006, PMID: 17429037

O'Hara T, Virag L, Varro A and Rudy Y. 2011. Simulation of the undiseased human cardiac ventricular action potential: model formulation and experimental validation. PLoS Computational Biology 7:e1002061. doi:10.1371/journal.pcbi.1002061, PMID: 21637795.

Okada Y, Ogawa S, Sadanaga T and Mitamura H. 1996. Assessment of reverse use-dependent blocking actions of class III antiarrhythmic drugs by 24-hour Holter electrocardiography. Journal of the American College of Cardiology 27:84-89. doi:10.1016/07351097(95)00424-6, PMID: 8522715.

Redfern WS, Carlsson L, Davis AS, Lynch WG, MacKenzie I, Palethorpe S, Siegl PKS, Strang I, Sullivan AT, Wallis R, Camm AJ and Hammond TG. 2003. Relationships between preclinical cardiac electrophysiology, clinical QT interval prolongation and torsade de pointes for a broad range of drugs: evidence for a provisional safety margin in drug development. Cardiovascular Research 58:32-45. doi:10.1016/S0008-6363(02)00846-5, PMID: 12667944.

Roden DM. 2000. Acquired long QT syndromes and the risk of proarrhythmia. Journal of Cardiovascular Electrophysiology 11:938-940. doi:10.1111/j.1540-8167.2000.tb00077.x PMID: 10969760.

Roden DM. 2008. Cellular basis of drug-induced torsades de pointes. British Journal of Pharmacology 154:1502-1507. doi:10.1038/bjp.2008.238, PMID: 18552874.

Sager PT, Gintant G, Turner JR, Pettit S and Stockbridge N. 2014. Rechanneling the cardiac proarrhythmia safety paradigm: a meeting report from the Cardiac Safety Research Consortium. American Heart Journal 167:292-300. doi:10.1016/j.ahj.2013.11.004, PMID: 24576511.

Sanguinetti MC, Jiang C, Curran ME and Keating MT. 1995. A mechanistic link between an inherited and an acquired cardiac arrhythmia: HERG encodes the IKr potassium channel. Cell 81:299-307. doi:10.1016/0092-8674(95)90340-2, PMID: 7736582.

Sanguinetti MC and Jurkiewicz NK. 1990. Two components of cardiac delayed rectifier K+ current. Differential sensitivity to block by class III antiarrhythmic agents. The Journal of General Physiology 96:195-215. doi:10.1085/jgp.96.1.195, PMID: 2170562.

Sanguinetti MC and Tristani-Firouzi M. 2006. hERG potassium channels and cardiac arrhythmia. Nature 440:463-469. doi:10.1038/nature04710, PMID: 16554806. 
Sato S, Zamami Y, Imai T, Tanaka S, Koyama T, Niimura T, Chuma M, Koga T, Takechi K, Kurata Y, Kondo Y, Izawa-Ishizawa Y, Sendo T, Nakura H and Ishizawa K. 2017. Metaanalysis of the efficacies of amiodarone and nifekalant in shock-resistant ventricular fibrillation and pulseless ventricular tachycardia. Scientific Reports 7:12683. doi:10.1038/s41598-017-13073-0, PMID: 28978927.

Satoh Y, Sugiyama A, Takahara A, Chiba K and Hashimoto K. 2004. Electropharmacological and proarrhythmic effects of a class III antiarrhythmic drug nifekalant hydrochloride assessed using the in vivo canine models. Journal of Cardiovascular Pharmacology 43:715-723, PMID: 15071360.

Sugiyama A. 2008. Sensitive and reliable proarrhythmia in vivo animal models for predicting drug-induced torsades de pointes in patients with remodelled hearts. British Journal of Pharmacology 154:1528-1537. doi:10.1038/bjp.2008.240, PMID: 18552873.

Surawicz B. 1989. Electrophysiologic substrate of torsade de pointes: dispersion of repolarization or early afterdepolarizations? Journal of the American College of Cardiology 14:172-184. doi:10.1016/0735-1097(89)90069-7, PMID: 2661626.

Takenaka K, Yasuda S, Miyazaki S, Kurita T, Sutani Y, Morii I, Daikoku S, Kamakura S and Nonogi H. 2001. Initial experience with nifekalant hydrochloride (MS-551), a novel class III antiarrhythmic agent, in patients with acute extensive infarction and severe ventricular dysfunction. Japanese Circulation Journal 65:60-62. doi:10.1253/jcj.65.60, PMID: 11153825 .

Tamargo J, Le Heuzey JY and Mabo P. 2015. Narrow therapeutic index drugs: a clinical pharmacological consideration to flecainide. European Journal of Clinical Pharmacology 71:549-567. doi:10.1007/s00228-015-1832-0, PMID: 25870032.

Tande PM, Bjornstad H, Yang T and Refsum H. 1990. Rate-dependent class III antiarrhythmic action, negative chronotropy, and positive inotropy of a novel Ik blocking drug, UK68,798: potent in guinea pig but no effect in rat myocardium. Journal of Cardiovascular Pharmacology 16:401-410, PMID: 1700210.

Thomsen MB, Volders PG, Stengl M, Spatjens RL, Beekman JD, Bischoff U, Kall MA, Frederiksen K, Matz J and Vos MA. 2003. Electrophysiological safety of sertindole in dogs with normal and remodeled hearts. Journal of Pharmacology and Experimental Therapeutics 307:776-784. doi:10.1124/jpet.103.052753, PMID: 12966159.

Toyama J, Kamiya K, Cheng J, Lee JK, Suzuki R and Kodama I. 1997. Vesnarinone prolongs action potential duration without reverse frequency dependence in rabbit ventricular muscle by blocking the delayed rectifier K+ current. Circulation 96:3696-3703. doi:10.1161/01.CIR.96.10.3696, PMID: 9396473.

Tsumoto K, Kurata Y, Furutani K and Kurachi Y. 2017. Hysteretic Dynamics of Multi-Stable Early Afterdepolarisations with Repolarisation Reserve Attenuation: A Potential Dynamical Mechanism for Cardiac Arrhythmias. Scientific Reports 7:10771. doi:10.1038/s41598-017-11355-1, PMID: 28883639.

Vaughan Williams EM. 1992. Classifying antiarrhythmic actions: by facts or speculation. The Journal of Clinical Pharmacology 32:964-977. doi:10.1002/j.1552-4604.1992.tb03797.x, PMID: 1474169.

Weiss JN, Garfinkel A, Karagueuzian HS, Chen PS and Qu Z. 2010. Early afterdepolarizations and cardiac arrhythmias. Heart Rhythm 7:1891-1899. doi:10.1016/j.hrthm.2010.09.017, PMID: 20868774. 
Yamakawa Y, Furutani K, Inanobe A, Ohno Y and Kurachi Y. 2012. Pharmacophore modeling for hERG channel facilitation. Biochemical and Biophysical Research Communications 418:161-166. doi:10.1016/j.bbrc.2011.12.153, PMID: 22244872.

Zeng J, Laurita KR, Rosenbaum DS and Rudy Y. 1995. Two components of the delayed rectifier $\mathrm{K}+$ current in ventricular myocytes of the guinea pig type. Theoretical formulation and their role in repolarization. Circulation Research 77:140-152. doi:10.1161/01.RES.77.1.140, PMID: 7788872.

Zeng J and Rudy Y. 1995. Early afterdepolarizations in cardiac myocytes: mechanism and rate dependence. Biophysical Journal 68:949-964. doi:10.1016/S0006-3495(95)80271-7, PMID: 7538806.

Zhou Z, Gong Q, Ye B, Fan Z, Makielski JC, Robertson GA and January CT. 1998. Properties of HERG channels stably expressed in HEK 293 cells studied at physiological temperature. Biophysical Journal 74:230-241. doi:10.1016/S0006-3495(98)77782-3, PMID: 9449325. 


\section{Figures:}

\section{Figure 1. Cardiac APs induce hERG facilitation with nifekalant.}

(A, B) Representative cell currents from hERG channels in Xenopus oocytes evoked by a test pulse from holding potential of $-90 \mathrm{mV}$ to $-50 \mathrm{mV}$ before and after AP stimulation ( $1 \mathrm{~Hz}, 20 \times$, AP waveform is the same as Figure $2 \mathrm{H}$ ) in the presence of $30 \mu \mathrm{M}$ nifekalant. (C) AP-facilitation relation. The fraction of facilitation induced by repeating APs are normalized to the fraction induced by the $+60 \mathrm{mV}$ conditioning pulse. Experimental data are means $\pm \operatorname{SEM}(n=8-15)$. The curve fit indicates exponential increase in the facilitation fraction by AP stimulation (Facilitation $=1.02-0.70 \cdot \exp (-(\# \mathrm{APs}) / \tau), \tau=$ $5.45 \pm 0.02)$.

\section{Figure 2. Experimental and simulated macroscopic hERG/I $I_{\mathrm{Kr}}$ currents as modified by} nifekalant.

(A-C) The macroscopic $\mathrm{hERG} / \mathrm{I}_{\mathrm{Kr}}$ currents in response to voltage-clamp pulses from -80 $\mathrm{mV}$ to $+60 \mathrm{mV}$ in $10 \mathrm{mV}$ increments from a holding potential of $-80 \mathrm{mV}$; representative traces (A), the relationship between membrane voltage and the steady-state current amplitude and membrane voltage (B) and the tail current amplitude (activation curve) (C). Experimental data are means $\pm \operatorname{SEM}(n=11)$. (D-G) Simulated effects of nifekalant on hERG/ $/ I_{\mathrm{Kr}}$ currents. The model assumes two populations of channels, with or without facilitation effect by nifekalant $(100 \mathrm{nM})$. The $V_{1 / 2}$ of activation for the facilitated fraction of channel was $\sim-31 \mathrm{mV}$, almost $26 \mathrm{mV}$ negative to that of control channel (see also Materials and Methods). (D, E) The macroscopic hERG/ $/ I_{\mathrm{Kr}}$ currents in response to voltage-clamp pulses from $-80 \mathrm{mV}$ to $-30 \mathrm{mV}$ (D) or $+30 \mathrm{mV}$ (E). (F, G) The relationship between the tail current amplitude and membrane voltage before $(\mathbf{F})$ and $\operatorname{after}(\mathbf{G})$ the induction of facilitation effect. Experimental data are means $\pm \operatorname{SEM}(n=$ 5). (H) The macroscopic $h E R G / I_{\mathrm{Kr}}$ currents in response to cardiac AP. In D-H, black, red, and green solid lines indicate with control, block with facilitation, and conventional block (block without facilitation), respectively. Under the condition in the block with facilitation, $I_{\mathrm{Kr}}$ comprises two fractions of $I_{\mathrm{Kr}}$ (see also main text), i.e., facilitated and unfacilitated fractions of $I_{\mathrm{Kr}}$. In $\mathbf{G}$ right panel, orange and cyan dashed lines represent 
the facilitated and unfacilitated fractions of $I_{\mathrm{Kr}}$, respectively. Experimental data are means $\pm \operatorname{SEM}(n=6-11)$.

\section{Figure 3. Effect of $I_{\mathrm{Kr}}$ facilitation on cardiac AP.}

(A) Simulated APs in an endocardial ventricular myocyte, (B) $I_{\mathrm{Kr}},(\mathbf{C})$ activation state values for unfacilitated $\left(x_{\mathrm{r} 1}\right)$ and facilitated $\left(x_{\mathrm{r} 2}\right)$ fractions of $I_{\mathrm{Kr}}$, and (D) $I_{\mathrm{K} 1}$ during APs with $100 \mathrm{nM}$ nifekalant. Through $\mathbf{A}$ to $\mathbf{D}$, black, green, and magenta solid lines indicate with control, block with facilitation, and conventional block (block without facilitation), respectively. Roman numerals above $\mathbf{A}$ indicate the phases of the AP in the case of with facilitation. a.u. indicates arbitrary unit.

Figure 4. Frequency-dependent effect of nifekalant on APD.

Increases in simulated $\mathrm{APD}_{90}$ from the control condition in the heart failure model with $100 \mathrm{nM}$ nifekalant (40\% $I_{\mathrm{Kr}}$ block) (A) and 50\% $I_{\mathrm{Kr}}$ block $(\mathbf{C})$ at each stimulation frequency. Effects of $I_{\mathrm{Kr}}$ block and facilitation on the APs in the heart failure model at various simulation frequency with $100 \mathrm{nM}$ nifekalant (40\% $I_{\mathrm{Kr}}$ block) (B) and 50\% $I_{\mathrm{Kr}}$ block (D).

\section{Figure 5. The effect of $I_{\mathrm{Kr}}$ facilitation on proarrhythmic risk.}

(A-C) The steady-state AP trains with 40\% (A), 50\% (B), and 55\% $I_{\mathrm{Kr}}$ block (C) in heart failing model with and without facilitation. (D) Effect of $I_{\mathrm{Kr}}$ block and facilitation on the APD and the development of EADs in heart failure model. Asterisk, dagger, and double-dagger indicate the conditions of in A-C, respectively. Sections and pipes indicate the upper limits of $I_{\mathrm{Kr}}$ block where APs ware normally terminated. In the bottom panel of $\mathbf{B}, \mathrm{AL}(\mathrm{EAD})$, alternated $\mathrm{EAD}$ (thin dashed trace); EAD, periodic EAD (bold dashed trace), see also main text.

Figure 6. Ionic mechanism of EAD development by $I_{\mathrm{Kr}}$ block and the influence of $I_{\mathrm{Kr}}$ facilitation.

Simulated APs and the changes in the membrane potential $\left(V_{\mathrm{m}}\right)(\mathbf{A}, \mathbf{B})$, L-type $\mathrm{Ca}^{2+}$ channels current, $I_{\mathrm{CaL}}(\mathbf{C})$, the net ionic current, $I_{\text {net }}(\mathbf{D})$, and $I_{\mathrm{Kr}}(\mathbf{E})$ during APs with 55\% $I_{\mathrm{Kr}}$ block in heart failing model. Each simulation of block (Left) and facilitation (Right) was started from same initial values. 


\section{Figure 7. Effect of $I_{\mathrm{Kr}}$ facilitation on the safety window of nifekalant.}

The changes in the membrane potential $\left(V_{\mathrm{m}}\right)$ (upper) and $I_{\mathrm{Kr}}$ (lower) in the presence of various concentrations of nifekalant in normal, non-failing model. The therapeutic dose is set as the concentration that prolongs $\mathrm{APD}_{90}$ by $500 \mathrm{~ms}$ and effectively suppresses the ectopic excitation (see also Supplemental Figure 5). 2.74× indicates 2.74 times higher drug concentration compared with its therapeutic dose $(1 \times)$. Green and magenta lines indicate $I_{\mathrm{Kr}}$ block with and without facilitation, respectively. Roman numerals at the bottom indicate the phases of the AP with facilitation.

\section{Supplemental Figure:}

\section{Supplemental Figure 1. Voltage dependence of activation and fast and slow deactivation} time constants.

(A) Time constant for activation. Squares indicate activation time constant values estimated from experimental data in Figure $2 \mathrm{~A}$, and solid and dashed lines represent fast $\left(\tau_{\mathrm{xr}, \text { fast }}\right)$ and slow $\left(\tau_{\mathrm{xr}, \text { slow }}\right)$ time constants convergences, respectively. (B-D) Time constants for deactivation. Tail current deactivation was examined from $-90 \mathrm{mV}$ to -30 $\mathrm{mV}(\mathbf{B})$ and was fitted by a standard double-exponential equation (c and $\mathbf{d})$. Points and circles represent fast (C) and slow (D) deactivation time constant values estimated from experimental data, respectively. The solid and dashed lines indicate the estimated $\tau_{\mathrm{xr} \text {,fast }}$ and $\tau_{\mathrm{xr} \text {,slow}}$, respectively. (E-F) Time constants as a function of membrane potential; fast (E) and slow (F) time constants for the activation and deactivations.

\section{Supplemental Figure 2. Concentration-dependent block and facilitation of nifekalant.}

(A) The relationship between the simulated tail current amplitude and membrane voltage at the indicated drug concentrations in the block with facilitation condition. Black and green lines indicate with control and block with facilitation, respectively. Orange and blue lines represent the facilitated and unfacilitated fractions of $I_{\mathrm{Kr}}$ in the block with facilitation condition, respectively. (B) Gray squares and gray dashed lines represent experimental concentration-dependent block and the fitted curves with Hill equation, respectively. Data are means $\pm \operatorname{SEM}(n=3-6)$. Open circles indicate the simulated concentration-dependent block. a.u. indicates arbitrary unit. 


\section{Supplemental Figure 3. Frequency-dependent effect of nifekalant on APD in non-failing} heart model.

Effects of $I_{\mathrm{Kr}}$ block and facilitation on the APs in the normal, non-failing model at various simulation frequency with $100 \mathrm{nM}$ nifekalant.

\section{Supplemental Figure 4. The effect of $I_{\mathrm{Kr}}$ facilitation on proarrhythmic risk in non-failing heart model.}

Effect of $I_{\mathrm{Kr}}$ block and facilitation on the APD and the development of EADs in normal, non-failing model (A). Asterisks indicate the conditions of in B. Symbols (daggers and double-daggers) indicate the upper limits of $I_{\mathrm{Kr}}$ block where APs ware normally terminated. (B) The steady-state AP trains with $86 \% I_{\mathrm{Kr}}$ block in non-heart failure model with and without facilitation. Representations and symbols are the same as in Figure 5.

\section{Supplemental Figure 5. The antiarrhythmic effect of $I_{K r}$ facilitation.}

Simulation of block and facilitation effect on the re-excitation by the second stimulation (S2) in endocardial ventricular myocyte model with the prolonged APD 90 by $500 \mathrm{~ms}$. AP responses to the S2 stimuli applied at the time indicated. 
Table 1. Effects of nifekalant on hERG channels

\begin{tabular}{|c|c|c|}
\hline \multicolumn{3}{|l|}{ Dose-response } \\
\hline & $\mathrm{IC}_{50} / \mathrm{EC}_{50}(\mathrm{nM})$ & hill coefficient \\
\hline Block & $144.92 \pm 16.00$ & 1.15 \\
\hline Facilitation & $92.84 \pm 7.71$ & 1.50 \\
\hline \multicolumn{3}{|c|}{ Shift of the hERG activation curve by facilitation effect } \\
\hline & \multicolumn{2}{|l|}{$\Delta \mathrm{V} 1 / 2(\mathrm{mV})$} \\
\hline HEK293 stably-expressing hERG & \multicolumn{2}{|l|}{-26.5} \\
\hline HEK293 transiently-expressing hERG & \multicolumn{2}{|l|}{-24.9} \\
\hline $\begin{array}{l}\text { HEK293 transiently-expressing hERG with } \\
\text { KCNE2 }\end{array}$ & \multicolumn{2}{|l|}{-24.4} \\
\hline X. Oocyte transiently-expressing hERG & \multicolumn{2}{|l|}{$-26.6(2)$} \\
\hline
\end{tabular}




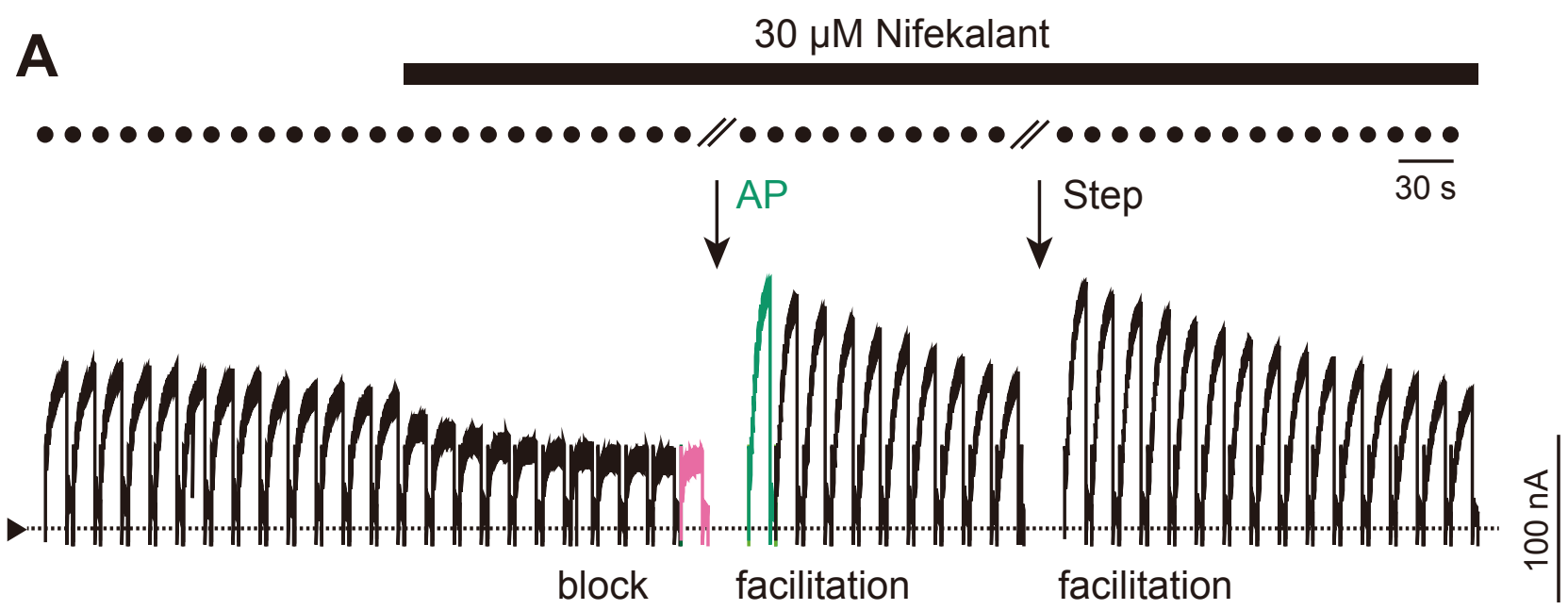

B

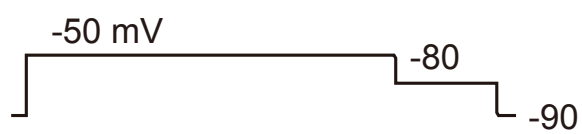

nifekalant block with AP-induced facilitation

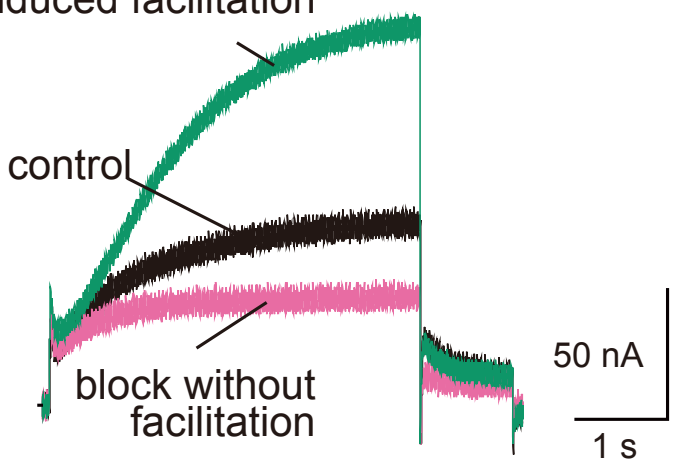

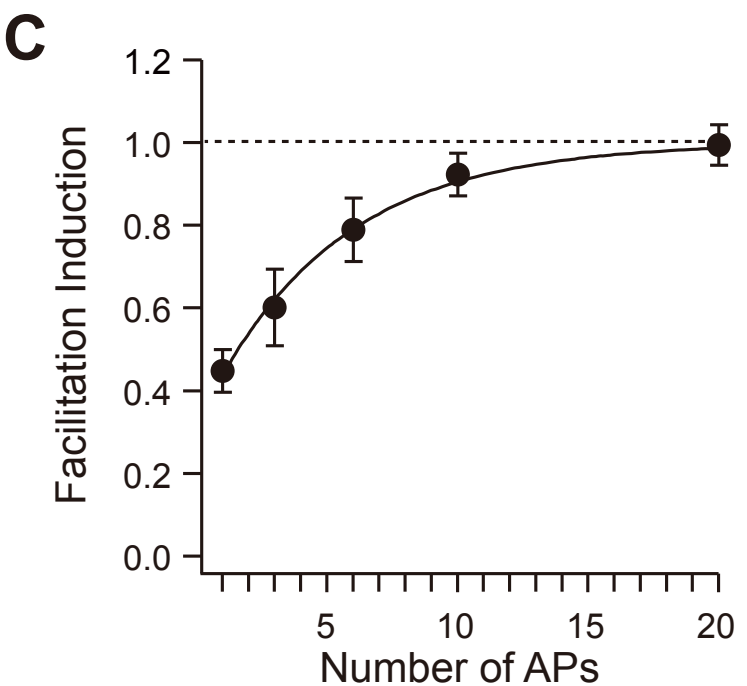

Figure 1. Cardiac APs induce hERG facilitation with nifekalant. 


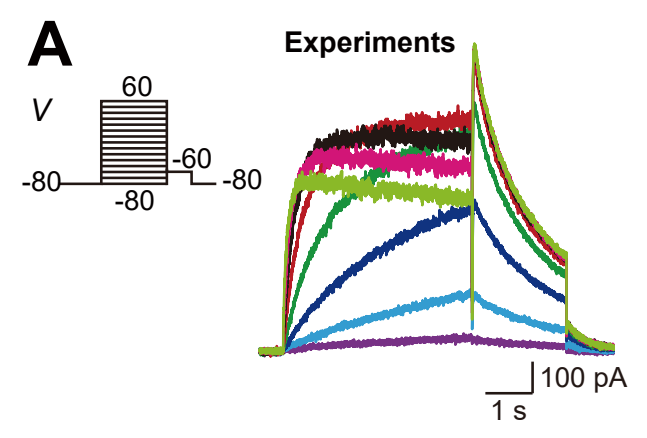

D Experiments Simulations
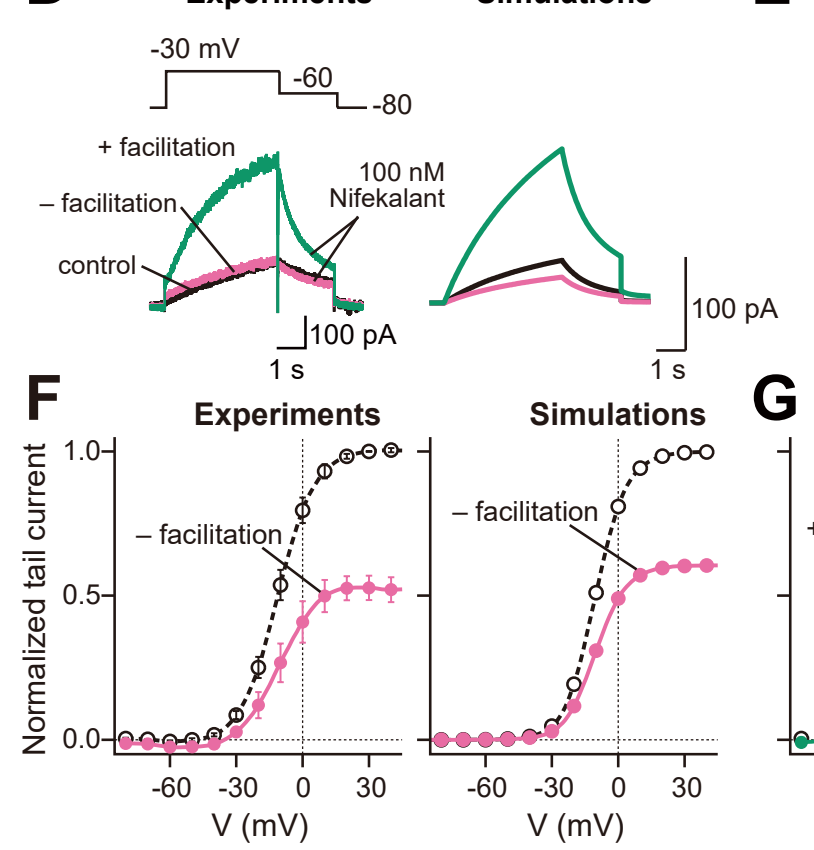

$\mathbf{G}$
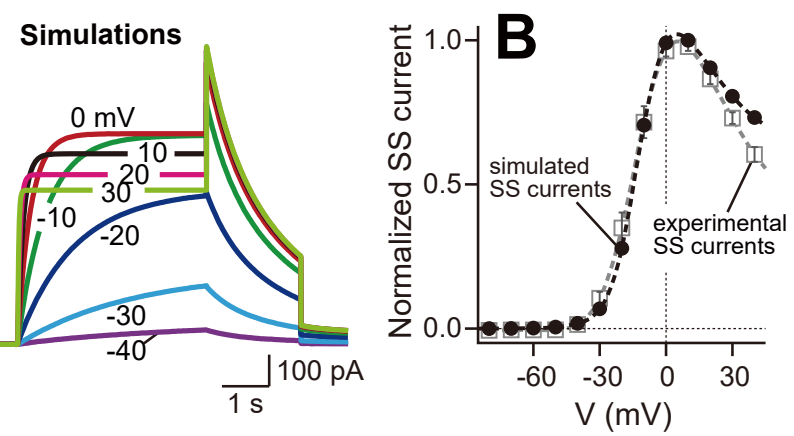

E Experiments

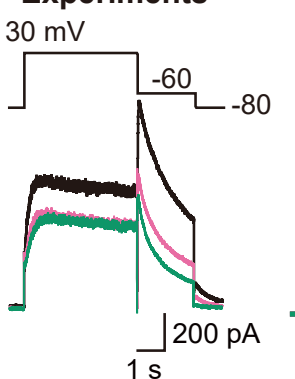

Simulations
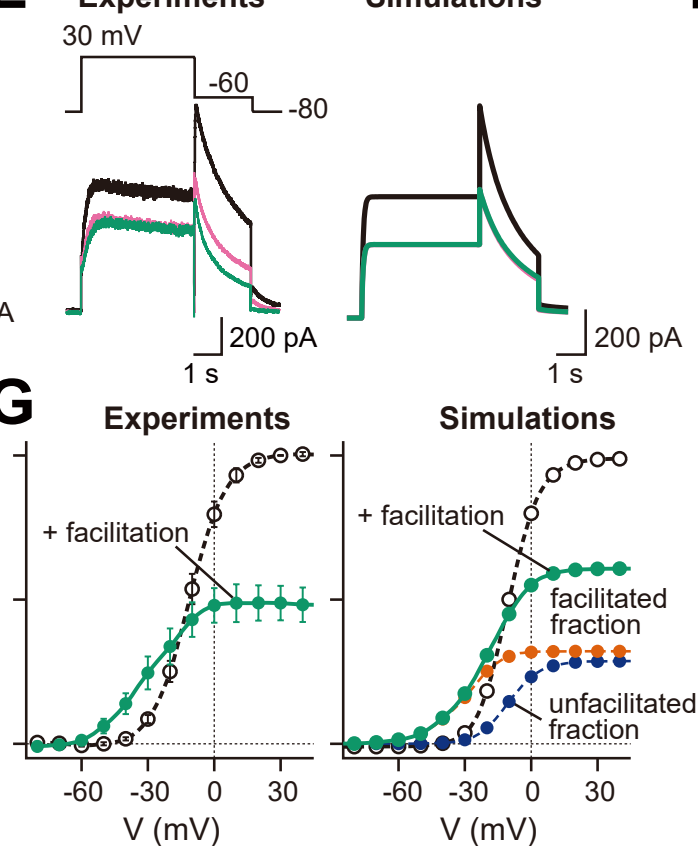

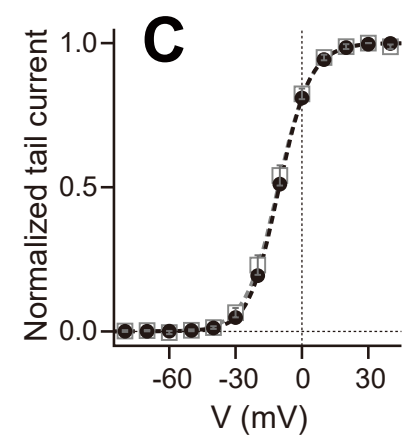

H
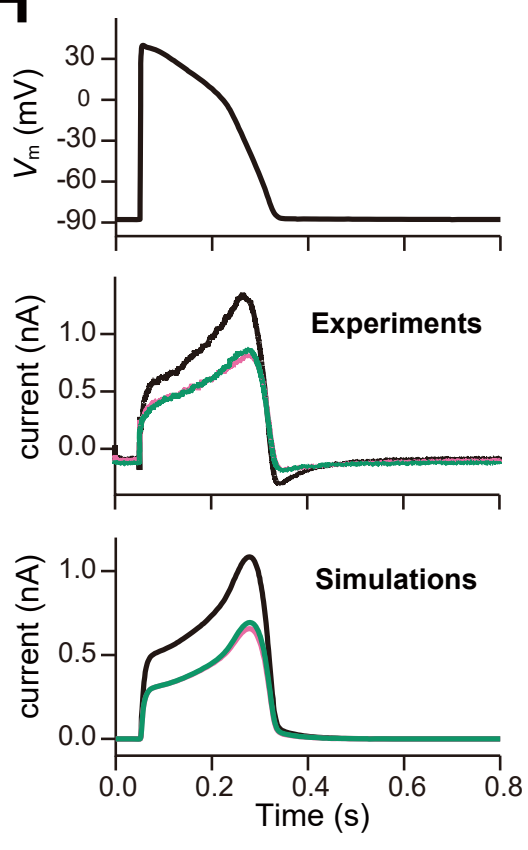

Figure 2. Experimental and simulated macroscopic $h E R G / I_{\mathrm{Kr}}$ currents as modified by nifekalant. 


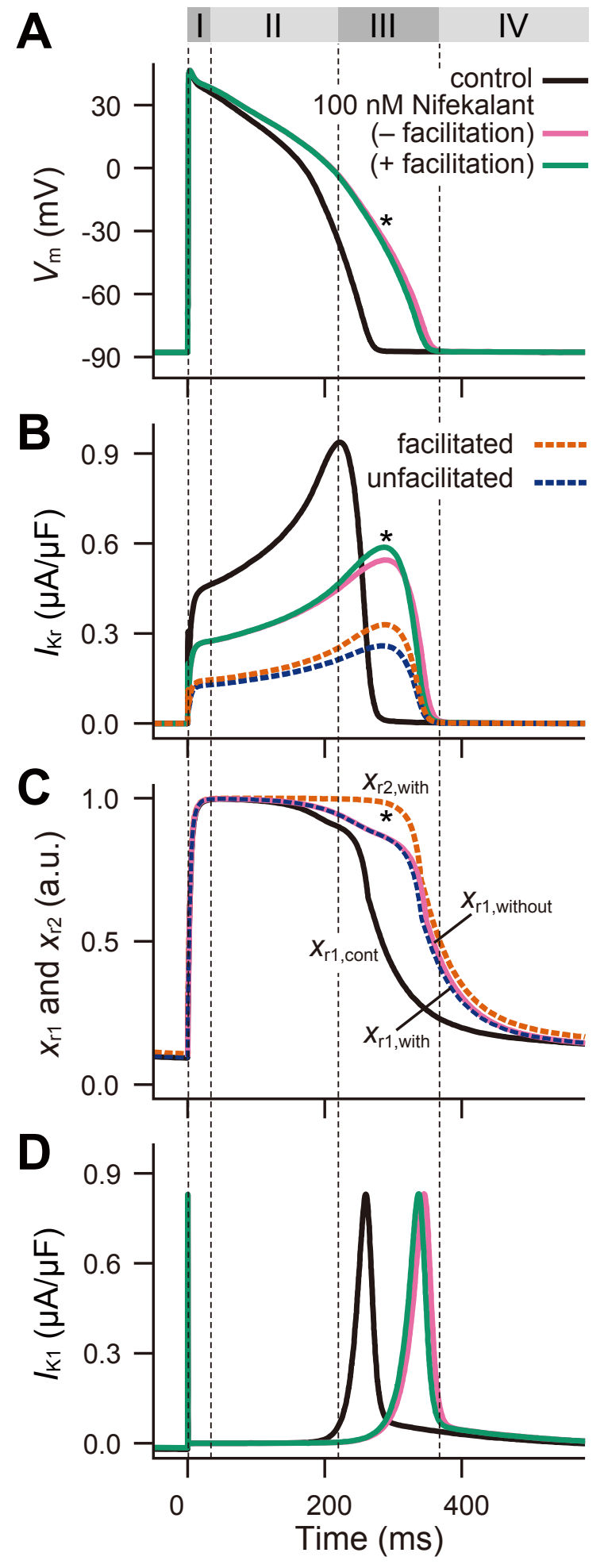

Figure 3. Effect of $I_{\mathrm{Kr}}$ facilitation on cardiac AP. 

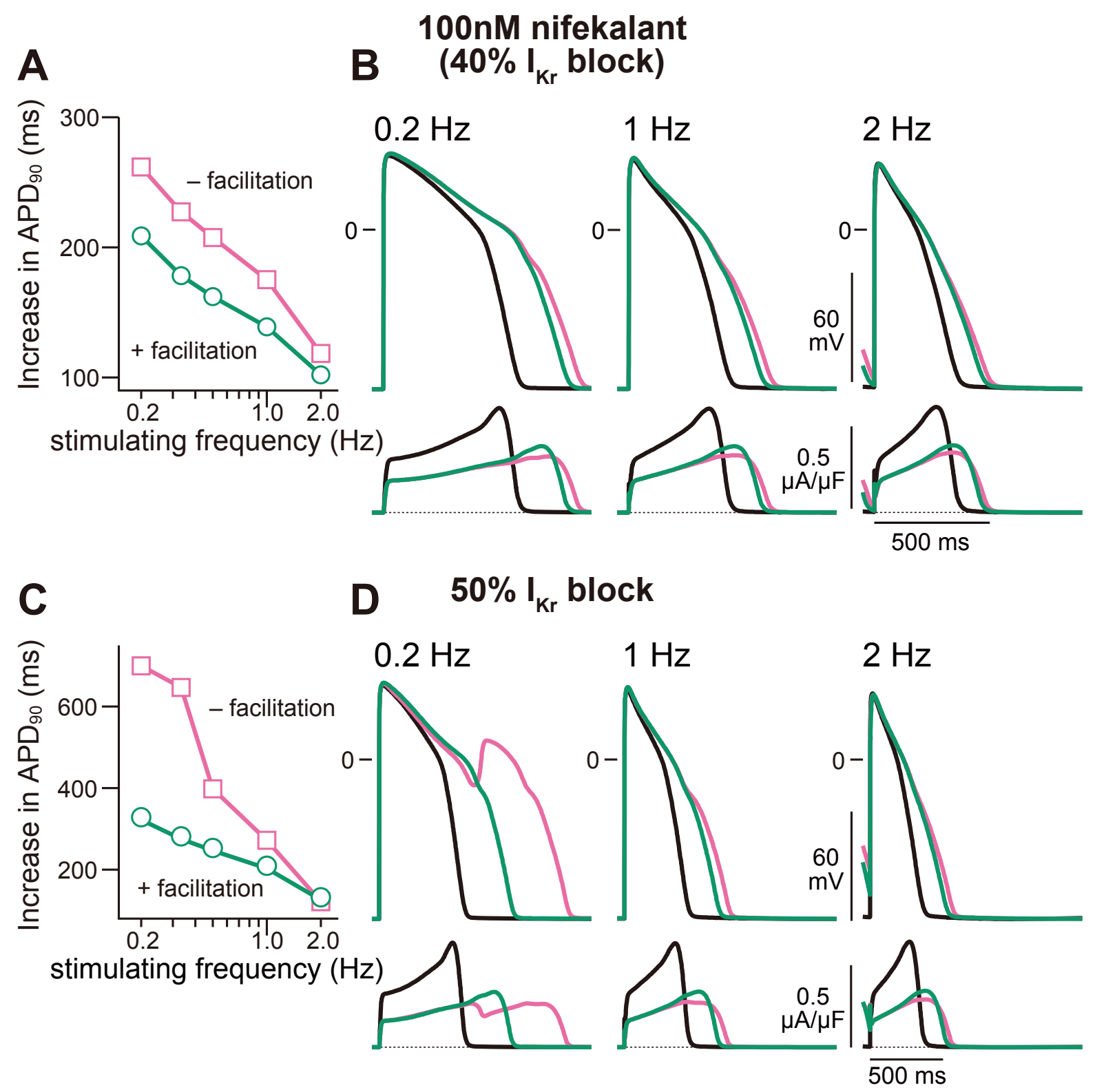

Figure 4. Frequency-dependent effect of nifekalant on APD. 


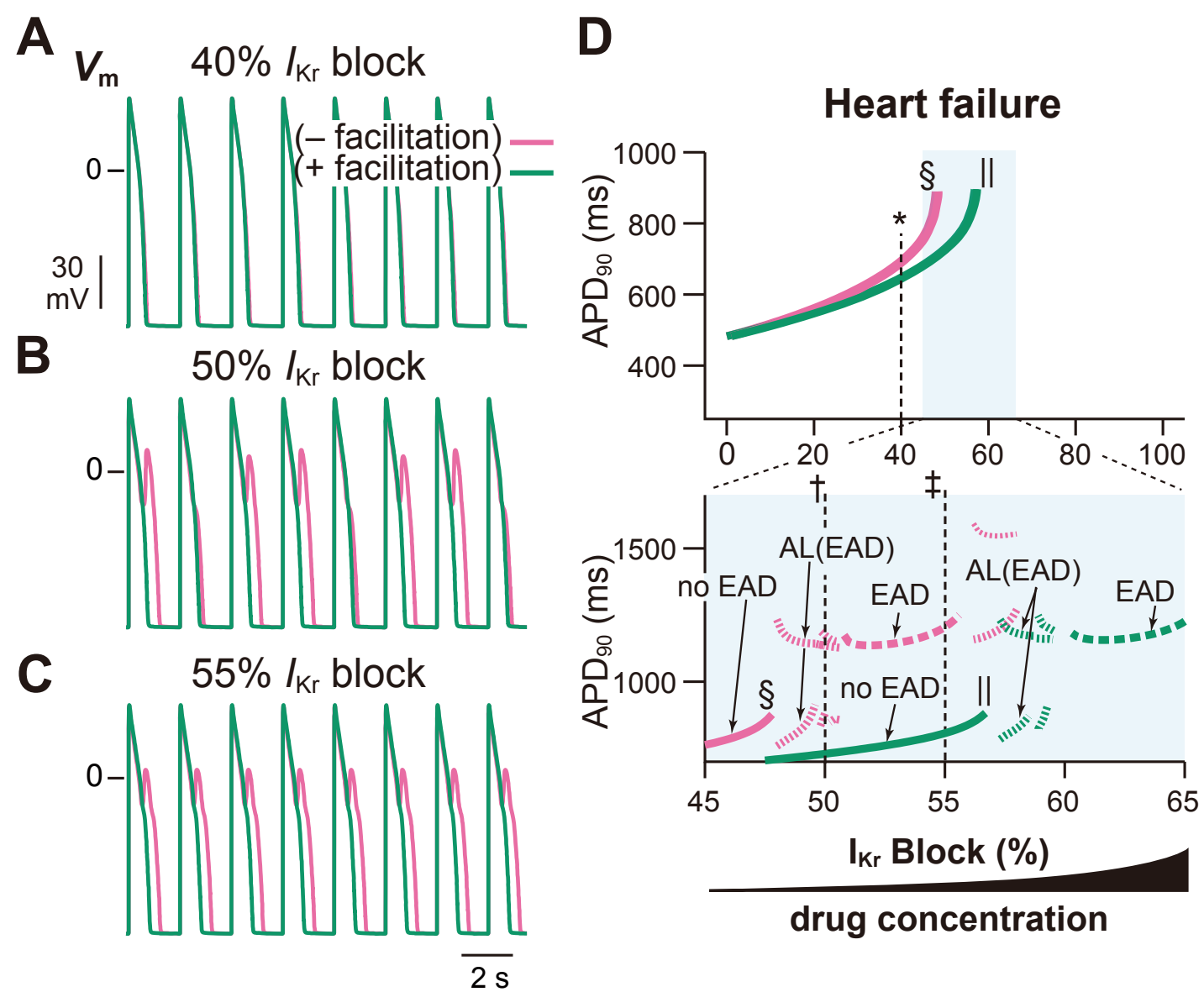

Figure 5. The effect of $I_{\mathrm{Kr}}$ facilitation on proarrhythmic risk. 


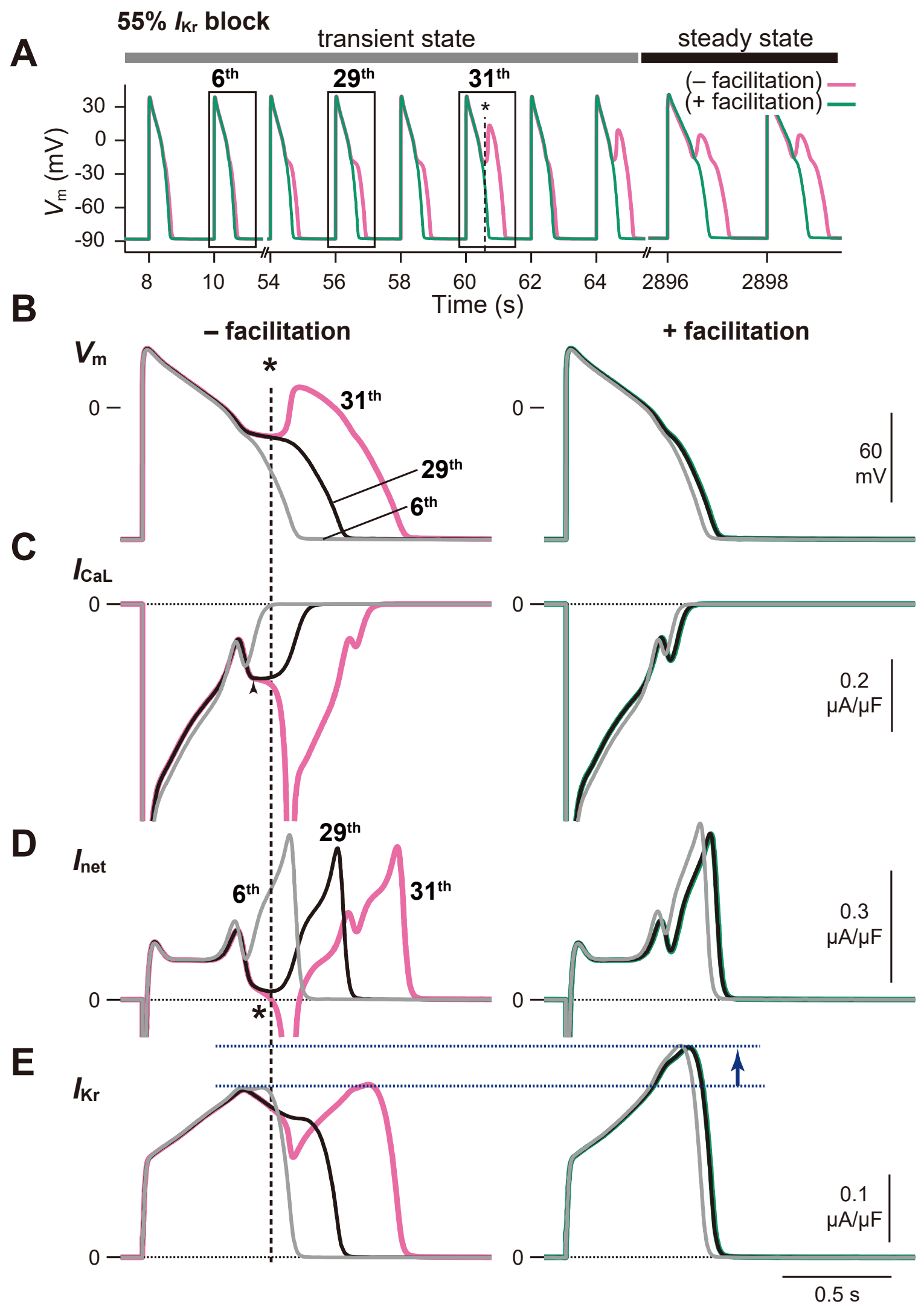

Figure 6. Ionic mechanism of EAD development by $I_{\mathrm{Kr}}$ block and the influence of $I_{\mathrm{Kr}}$ facilitation. 

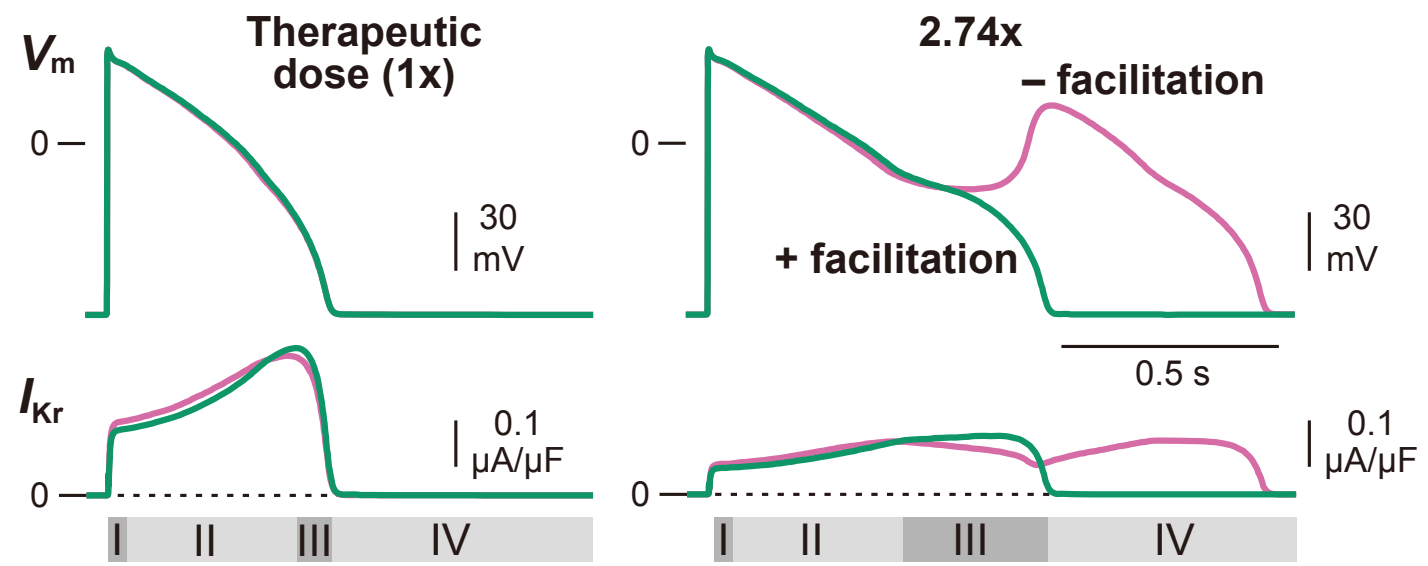

Figure 7. Effect of $I_{\mathrm{Kr}}$ facilitation on the safety window of nifekalant. 

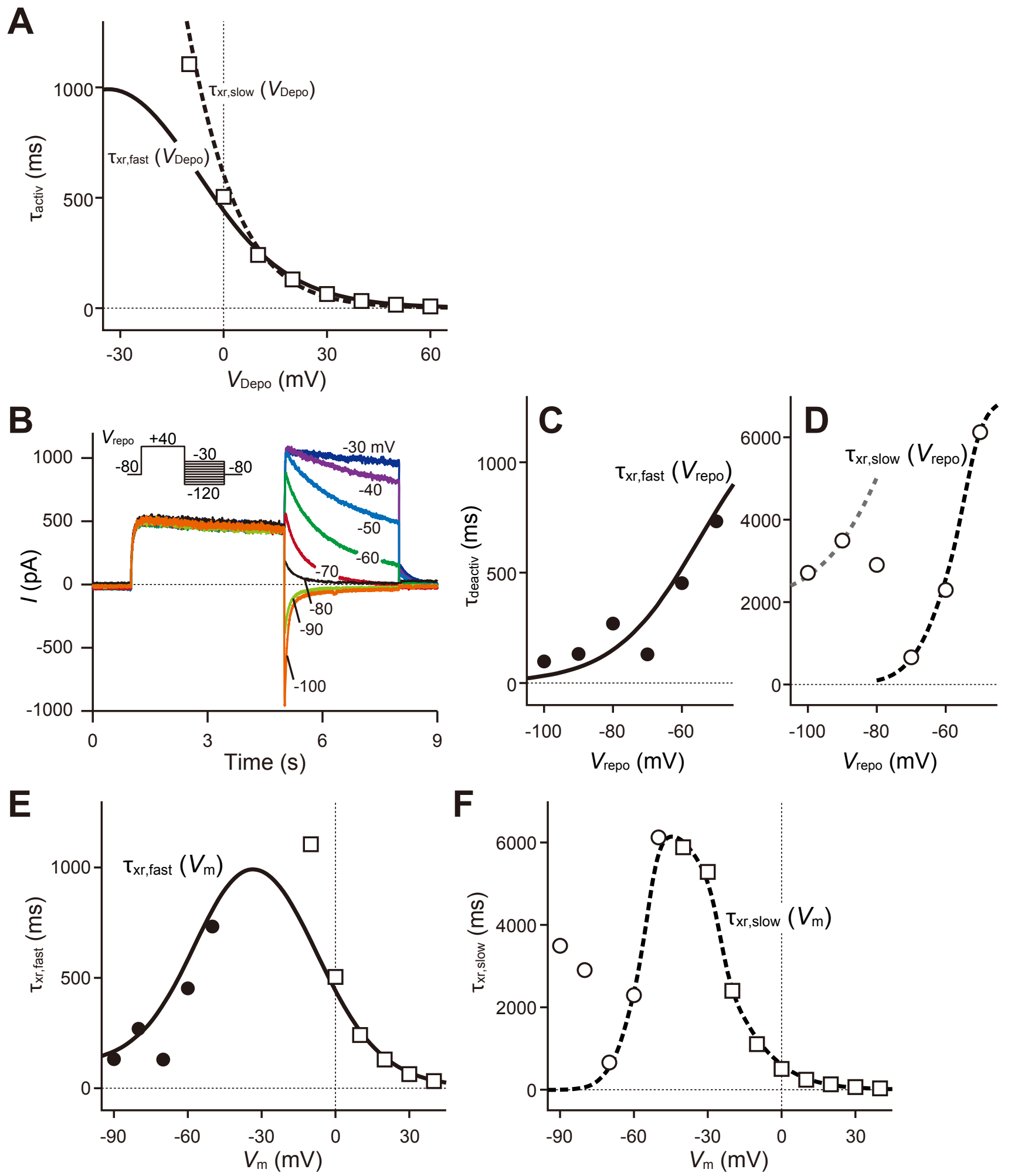

Supplemental Figure 1. Voltage dependence of activation and fast and slow deactivation time constants. 

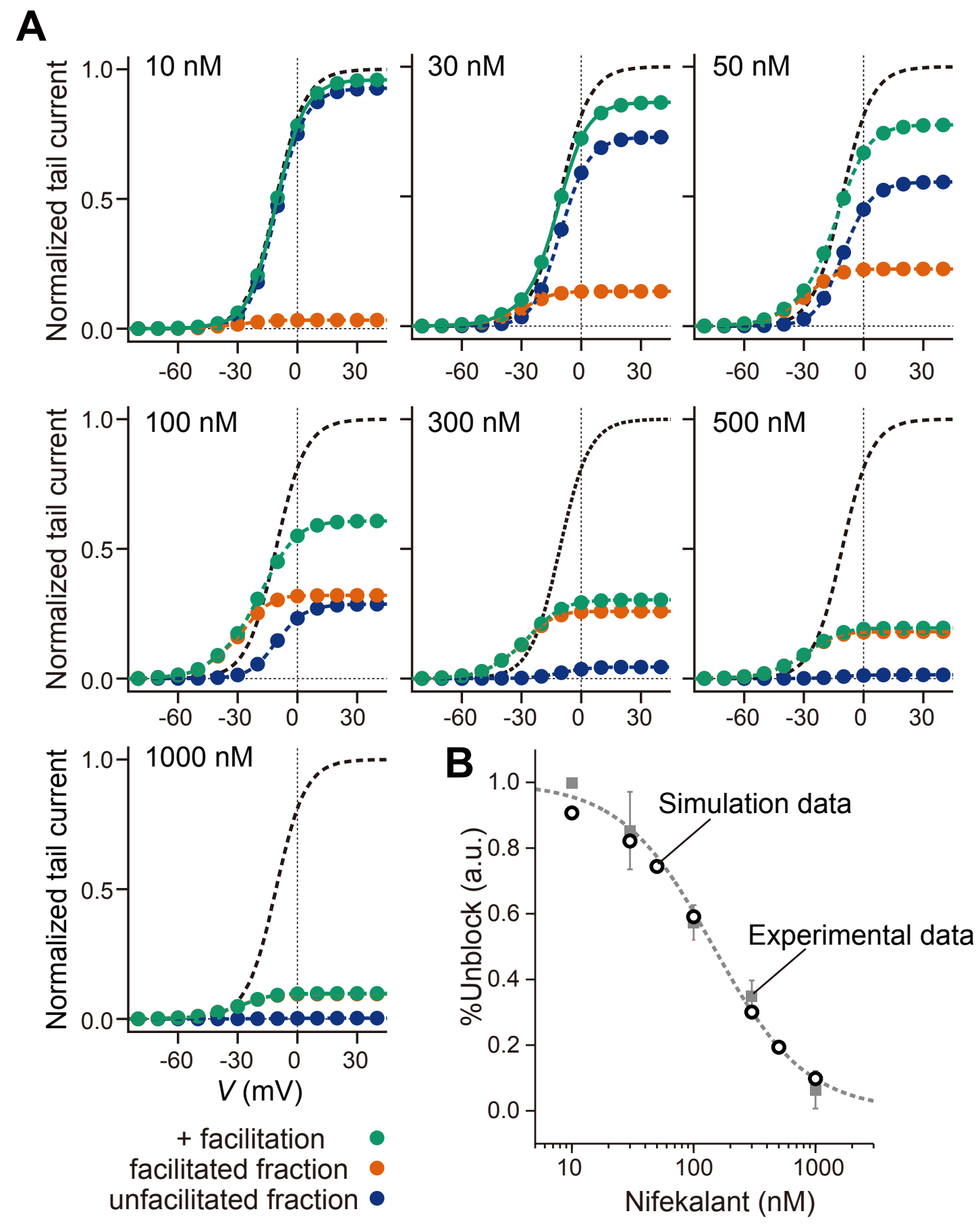

Supplemental Figure 2. Concentration-dependent block and facilitation of nifekalant. 

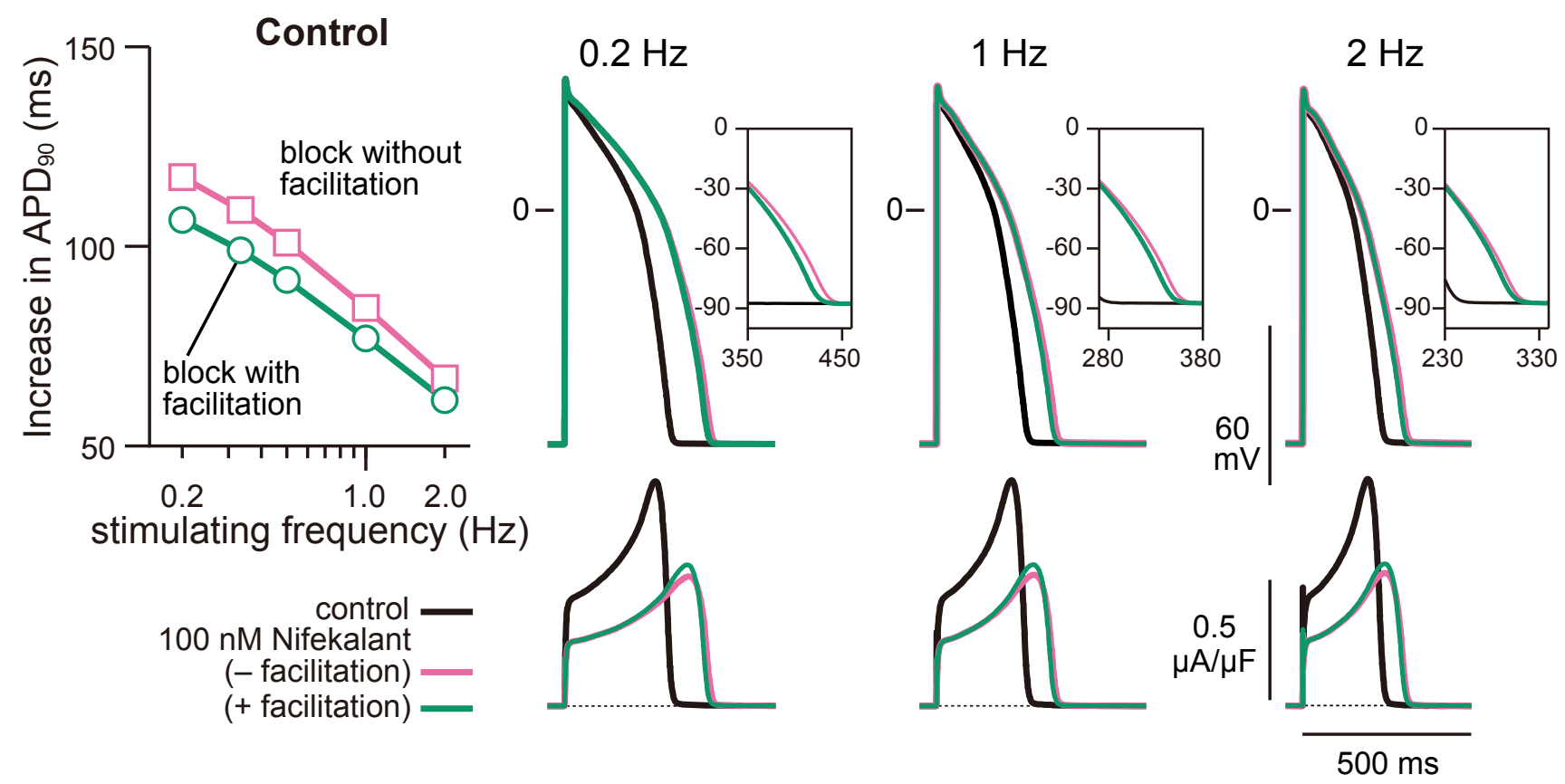

Supplemental Figure 3. Frequency-dependent effect of nifekalant on APD in non-failing heart model. 


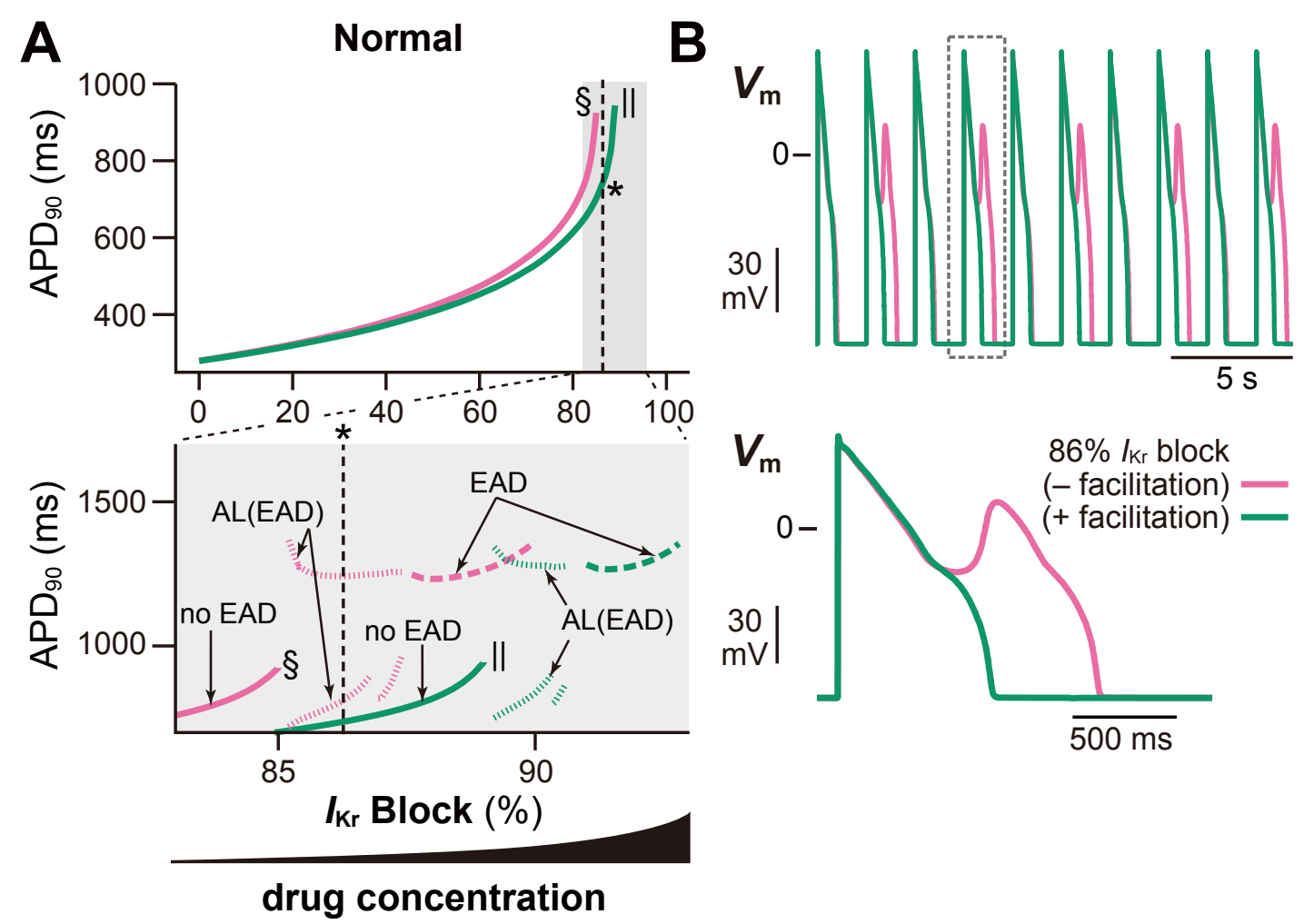

Supplemental Figure 4. The effect of $I_{\mathrm{Kr}}$ facilitation on proarrhythmic risk in non-failing heart model. 

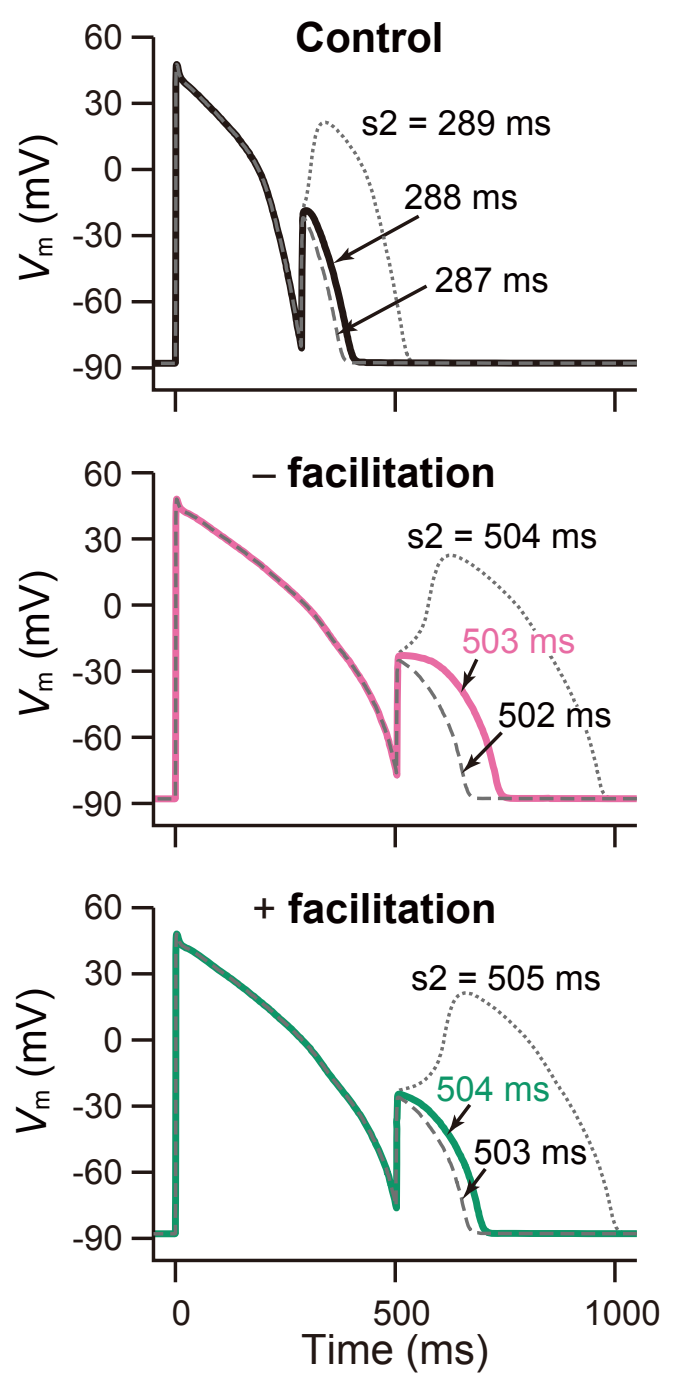

Supplemental Figure 5. The antiarrhythmic effect of $I_{K \mathrm{r}}$ facilitation. 